\title{
Brinkmann Model and Double Penalization Method for the Flow Around a Porous Thin Layer
}

\author{
Gilles Carbou
}

Mathématiques Appliquées de Bordeaux, UMR 5466 et Université Bordeaux 1, 351 cours de la Libération, 33405 Talence cedex, France.

\begin{abstract}
: in this paper we study a penalization method used to compute the flow of a viscous fluid around a thin layer of porous material. Using a BKW method, we perform an asymptotic expansion of the solution when a little parameter, measuring the thickness of the thin layer and the inverse of the penalization coefficient, tends to zero. We compare then this numerical method with a Brinkman model for the flow around a porous thin layer.
\end{abstract}

Subject Classification: 35B25, 76D05, 76D10

Keywords: Navier-Stokes equations, BKW method, penalization, porous material, thin layer.

\section{Introduction}

The penalization methods are used since the former works of Peskin [16], [17], about twenty years ago, in order to compute the flow of an incompressible fluid in a complex geometry. The aim is to avoid body-fitted unstructured mesh in order to use accurate and fast spectral methods [14] or finite volumes approximations on cartesian meshes [13]. In [2], [3], [5], [6], [7], [10] [13], [18], the different authors add a penalization term on the velocity defined on the volume of the obstacle.

In this paper we study a penalization method used by C. H. Bruneau and I. Mortazavi in [8] in order to compute the flow of a viscous fluid around a ground vehicle surrounded by a thin layer of porous material.

We consider $\mathcal{O}$ a $\mathcal{C}^{\infty}$-bounded domain of $\mathbb{R}^{3}$ and $\Omega$ a $\mathcal{C}^{\infty}$-open subset of $\mathcal{O}$ such that $\bar{\Omega} \subset \mathcal{O}$. We denote $\Omega_{\text {ext }}=\mathcal{O} \backslash \bar{\Omega}$ and $\Gamma=\partial \Omega$. We fix $\kappa>0$.

For $\varepsilon>0$, we set

- $\omega_{\varepsilon}=\{x \in \Omega, \quad 0<\operatorname{dist}(x, \Gamma)<\kappa \varepsilon\}$

- $\Gamma_{\varepsilon}=\{x \in \Omega, \quad \operatorname{dist}(x, \Gamma)=\kappa \varepsilon\}$

- $\Omega_{\varepsilon}=\Omega \backslash \overline{\omega_{\varepsilon}}$

- $\Omega_{f l u}^{\varepsilon}=\Omega_{e x t} \cup \Gamma \cup \omega_{\varepsilon}$ 
We will perform for both problems (1.1) and (1.2) an asymptotic expansion of the solutions when $\varepsilon$ goes to zero. For the Brinkmann model (1.2) we perform in the thin layer $\omega_{\varepsilon}$ a rescaling in order to work in a fixed domain with an equation depending of $\varepsilon$. For the double penalization method (1.1), we treat the thin layer as for (1.2) and we couple the asymptotic expansion of the solution in the thin layer with the boundary layer that appears in the obstacle $\Omega_{\varepsilon}$. These asymptotic expansions are obtained with a BKW method.

With these two asymptotic expansions, we will compare both models. We will prove that the solution of (1.1) is similar to the solutions of (1.2) around a porous thin layer of thickness $(1+\kappa) \varepsilon$, that is the layer thickness for the Brinkmann model is different to the layer thickness for the numerical process.

Let us describe more precisely our different results.

Let $U^{0}$ be a regular solution for the flow around the obstacle $\Omega$ with initial data $U^{0}(t=0)=u_{0}$, that is

$$
\left\{\begin{array}{l}
\frac{\partial U^{0}}{\partial t}-\Delta U^{0}+\left(U^{0} \cdot \nabla\right) U^{0}+\nabla p^{0}=f \text { in }\left[0, T^{*}\left[\times \Omega_{e x t}\right.\right. \\
\operatorname{div} U^{0}=0 \text { in }\left[0, T^{*}\left[\times \Omega_{e x t}\right.\right. \\
U^{0}=0 \text { in }\left[0, T^{*}\left[\times \partial \Omega_{e x t}\right.\right. \\
U^{0}(t=0, x)=u_{0}(x) \text { in } \Omega_{e x t}
\end{array}\right.
$$

with the following regularity property, for $i:=0 . .2$,

$$
\left\{\begin{array}{l}
\frac{\partial^{i} U^{0}}{\partial t^{i}} \in L^{\infty}\left(0, T ; H^{5-2 i}\left(\Omega_{e x t}\right)\right) \cap L^{2}\left(0, T ; H^{7-2 i}\left(\Omega_{e x t}\right)\right), \\
\frac{\partial^{i} p^{0}}{\partial t^{i}} \in L^{\infty}\left(0, T ; H^{4-2 i}\left(\Omega_{e x t}\right)\right) \cap L^{2}\left(0, T ; H^{6-2 i}\left(\Omega_{e x t}\right)\right) .
\end{array}\right.
$$

Remark 1.2 The existence of the regular solution for Navier Stokes equation is a quite classical result (see for example [4], [9] or [10])

We know in addition that $\frac{\partial U^{0}}{\partial n}$ i is tangent to $\Gamma$.

Furthermore, the time $T^{*}$ is the blow up time for the regular solution of (1.3). In particular, we know that $T^{*}=+\infty$ in the two dimensional case.

With classical technics we can prove the existence of weak solutions for the Brinkmann Model (see Leray's arguments in [15]). In the following theorem we perform an asymptotic expansion for a weak solution for Brinkmann model (1.2) with initial data $u^{\varepsilon}(t=0)=U^{0}(t=0)=u_{0}$ : 
Theorem 1.1 Let $U^{0}$ and $p^{0}$ the regular profile satisfying (1.3)-(1.4). Let $u^{\varepsilon}$ be weak solution of (1.2) with initial data $u^{\varepsilon}(t=0)=u_{0}$ in $\Omega_{e x t}$ and $u^{\varepsilon}(t=0)=0$ in $\omega_{\varepsilon}$. Then $u^{\varepsilon}$ satisfies the following asymptotic expansion:

$$
u^{\varepsilon}(t, x)=U^{0}(t, x)+\varepsilon U^{1}(t, x)+\varepsilon^{\frac{3}{2}} v_{\varepsilon}^{r}(t, x) \text { for } x \in \Omega_{e x t}
$$

where the profile $U^{1}:\left[0, T^{*}\left[\times \Omega_{e x t} \longrightarrow \mathbb{R}^{3}\right.\right.$ satisfies

$$
\left\{\begin{array}{l}
\frac{\partial U^{1}}{\partial t}-\Delta U^{1}+\left(U^{0} \cdot \nabla\right) U^{1}+\left(U^{1} \cdot \nabla\right) U^{0}+\nabla p^{1}=0 \quad \text { in }\left[0, T^{*}\left[\times \Omega_{e x t},\right.\right. \\
\operatorname{div} U^{1}=0 \quad \text { in }\left[0, T^{*}\left[\times \Omega_{\text {ext }},\right.\right. \\
U^{1}=-\kappa \frac{\partial U^{0}}{\partial n} \quad \text { on }\left[0, T^{*}[\times \Gamma,\right. \\
U^{1}=0 \quad \text { on }\left[0, T^{*}[\times \partial \mathcal{O},\right.
\end{array}\right.
$$

and where the remainder term $v_{\varepsilon}^{r}$ is bounded uniformely with respect to $\varepsilon$ in the space $L^{\infty}\left(0, T ; H^{1}\left(\Omega_{\text {ext }}\right)\right)$ that for all $T<T^{*}$,

We study now the numerical process (1.1). We will perform an asymptotic expansion of the solutions of (1.1). In the thin layer we describe the solution in the variables $(\sigma, z) \in \Gamma \times] 0, \kappa[$ where $\sigma=P(x)$ is the orthogonal projection of $x$ on $\Gamma$, and where $z=\frac{\varphi(x)}{\varepsilon}$ with $\varphi(x)=\operatorname{dist}(x, \Gamma)$. In the obstacle $\Omega_{\varepsilon}$, it appears a boundary layer described with the fast variable $\frac{\Psi(x)}{\varepsilon}$ where $\Psi(x)=\operatorname{dist}\left(x, \Gamma_{\varepsilon}\right)$.

Theorem 1.2 Let $U^{0}$ and $p^{0}$ be the regular solution of (1.3)-(1.4). Let $u^{\varepsilon}$ be a weak solution of Equation (1.1) with initial data $u^{\varepsilon}(t=0)=U^{0}(t=0)$ in $\Omega_{e x t}$ and $u^{\varepsilon}(t=0)=0$ in $\Omega$. Then $u^{\varepsilon}$ satisfies the following asymptotic expansion:

$$
u^{\varepsilon}(t, x)=\left\{\begin{array}{l}
U^{0}(t, x)+\varepsilon \mathbf{U}^{1}(t, x)+\varepsilon^{\frac{3}{2}} v_{\varepsilon}^{r}(t, x) \text { for } x \in \Omega_{e x t}, \\
\varepsilon \mathbf{V}^{1}\left(t, P(x), \frac{\varphi(x)}{\varepsilon}\right)+\varepsilon^{\frac{3}{2}} v_{\varepsilon}^{r}(t, x) \text { for } x \in \omega_{\varepsilon}, \\
\varepsilon \mathbf{W}^{1}\left(t, x, \frac{\Psi(x)}{\varepsilon}\right)+\varepsilon^{\frac{3}{2}} v_{\varepsilon}^{r}(t, x) \text { for } x \in \Omega_{\varepsilon},
\end{array}\right.
$$

where

- $\mathbf{U}^{1}:\left[0, T^{*}\left[\times \Omega_{\text {ext }} \longrightarrow \mathbb{R}^{3}\right.\right.$ satisfies 


$$
\left\{\begin{array}{l}
\frac{\partial \mathbf{U}^{1}}{\partial t}-\Delta \mathbf{U}^{1}+\left(U^{0} \cdot \nabla\right) \mathbf{U}^{1}+\left(\mathbf{U}^{1} \cdot \nabla\right) U^{0}+\nabla p^{1}=0 \text { in }\left[0, T^{*}\left[\times \Omega_{\text {ext }},\right.\right. \\
\operatorname{div} \mathbf{U}^{1}=0 \text { in }\left[0, T^{*}\left[\times \Omega_{\text {ext }},\right.\right. \\
\mathbf{U}^{1}=-(\kappa+1) \frac{\partial U^{0}}{\partial n} \text { on }\left[0, T^{*}[\times \Gamma,\right. \\
\mathbf{U}^{1}=0 \text { on }\left[0, T^{*}[\times \partial \mathcal{O} .\right.
\end{array}\right.
$$

- $\mathbf{V}^{1}$ is defined on $\left[0, T^{*}\left[\times \Gamma \times[0, \kappa]\right.\right.$ with values in $\mathbb{R}^{3}$,

- $\mathbf{W}^{1}$ is defined on $\left[0, T^{*}\left[\times \Omega_{\varepsilon} \times \mathbb{R}^{+}\right.\right.$with values in $R^{3}$,

- the remainder term $v_{\varepsilon}^{r}$ is bounded in $L^{2}\left(0, T ; H^{1}(\mathcal{O})\right) \cap L^{\infty}\left(0, T ; L^{2}(\mathcal{O})\right)$ for all $T<T^{*}$.

Remark 1.3 We note that the first terms $U^{1}$ and $\mathbf{U}^{1}$ given respectively by (1.5) and (1.7) are different.

Therefore in order to obtain a good approximation of the solutions of (1.2) we have to modify the numerical process (1.1) taking the porous layer thickness equal to $(\kappa-1) \varepsilon$.

Remark 1.4 In [10] we study another model for the flow around a porous thin layer and we perform for this model an asymptotic expansion compatible with the expansion obtained here for the Brinkmann model (1.2). The particularities of the present work are first the new Brinkmann model (see Remark 1.1) and that we couple for the study of (1.1) the thin layers methods with the boundary layers ones.

This paper is organized as follows.

In the second part, we briefly recall the geometrical tools used for the study of the thin layer $\omega_{\varepsilon}$, and we mention usefull analytical results.

In the third part, we study the Brinkmann model. We perform the asymptotic expansion of the solutions of (1.2) and we prove Theorem 1.1.

The last part is devoted to the proof of Theorem 1.2.

The proofs of Theorems 1.1 and 1.2 are based on the following method. In a first step we perform a formal BKW method, that is we assume that the solution admits an asymptotic expansion given in the theorem, and we plug this expansion in the equations. Identifying the different powers of $\varepsilon$ we obtain then equations characterizing the profiles in the asymptotic expansion. In a second step we prove the existence and the regularity of the profiles. In a last step, we define by difference the remainder term and we estimate it using rather classical variational estimates. This estimation gives a rigorous validation of the asymptotic expansion. 


\section{Preliminary results}

\subsection{Geometrical tools for the thin layers}

In order to describe the behaviour of the flow in the thin layer $\omega_{\varepsilon}$ we use technics developped in [9] and used in [19] in the framework of ferromagnetism.

We will write the equations in the thin layer using the coordinates $(\sigma, z)$ where $\sigma=P(x)$ is the projection of $x$ onto $\Gamma$ and $z$ is the distance between $x$ and $\Gamma$. We use these coordinates because we can easily rescale in the variable $z$ the equations and then work in the fixed domain $\Gamma \times[0, \kappa]$.

We use the parametrization of $\omega_{\varepsilon}$ defined by :

$$
\begin{aligned}
\Theta: \quad \Gamma \times] 0, \kappa \varepsilon[ & \longrightarrow \omega_{\varepsilon} \\
\sigma, z & \mapsto \sigma+z n(\sigma)
\end{aligned}
$$

Since $\Gamma=\partial \Omega$ is a regular compact surface of $\mathbb{R}^{3}$ without boundary, there exists $\eta_{0}>0$ such that for $\varepsilon<\eta_{0}, \Theta$ is a $\mathcal{C}^{\infty}$-diffeomorphism from $\left.\Gamma \times\right] 0, \kappa \varepsilon\left[\right.$ onto $\omega_{\varepsilon}$. Furthermore $\varphi$ and $P$ are regular on $\omega_{\eta_{0}}$ and

$$
\forall x \in \omega_{\eta_{0}}, \quad \nabla \varphi(x)=n(P(x)) .
$$

On the submanifold $\Gamma$ we can classicaly define the integrale and the differential operators $\nabla_{\Gamma}$, div $\Gamma$ and $\Delta_{\Gamma}$. Furthermore, $n$ is a map defined from $\Gamma$ with values in the unit sphere $S^{2}$ so for $\sigma \in \Gamma$, the differential $d n(\sigma)$ is a linear map from $T_{\sigma} \Gamma$ into $T_{n(\sigma)} S^{2}$ (where $T_{\sigma} \Gamma$ denote the tangent plane of $\Gamma$ at the point $\sigma)$. Since $T_{n(\sigma)} S^{2}=T_{\sigma} \Gamma$, we can consider $d n(\sigma)$ as an endomorphism of $T_{\sigma} \Gamma$.

Gradient : for $\tilde{v}: \Gamma \longrightarrow \mathbb{R}$, we define :

$$
\nabla_{\Gamma_{s}} \tilde{v}(\sigma)=(I d+s d n(\sigma))^{-1}\left(\nabla_{\Gamma} \tilde{v}(\sigma)\right)
$$

and if $u: \omega_{\eta_{0}} \longrightarrow \mathbb{R}$, denoting $\tilde{u}=u \circ \Theta$, we have :

$$
\nabla u(x)=\frac{\partial \tilde{u}}{\partial z}(P(x), \varphi(x)) n(P(x))+\left(\nabla_{\Gamma_{\varphi(x)}} \tilde{u}\right)(P(x), \varphi(x)) .
$$

Divergence Operator : let $\tilde{Y}: \Gamma \longrightarrow T \Gamma$ be a tangent vector field defined on $\Gamma$. For $s \in\left[0, \kappa \eta_{0}[\right.$ and $\sigma \in \Gamma$, we define:

$$
\operatorname{div}_{\Gamma_{s}} \tilde{Y}(\sigma)=\frac{1}{\gamma_{s}(\sigma)} \operatorname{div}_{\Gamma}\left[\gamma_{s}(I d+s d n)^{-1} \tilde{Y}\right](\sigma),
$$

where

$$
\gamma_{s}(\sigma)=\operatorname{det}(I d+s d n(\sigma))
$$

In addition if $Z: \omega_{\eta_{0}} \longrightarrow \mathbb{R}^{3}$, denoting $\widetilde{Z}=Z \circ \Theta$, we have

$$
\begin{aligned}
\operatorname{div} Z(x)= & \frac{\partial \widetilde{Z}_{N}}{\partial z}(P(x), \varphi(x))+G_{\varphi(x)}(P(x)) \widetilde{Z}_{N}(P(x), \varphi(x)) \\
& +\left(\operatorname{div}_{\Gamma_{\varphi(x)}} \widetilde{Z}_{T}\right)(P(x), \varphi(x)),
\end{aligned}
$$


where $\widetilde{Z}_{N}(\sigma, z)=(\widetilde{Z}(\sigma, z) \cdot n(\sigma))$ is the normal part of $\tilde{Z}$ and $\widetilde{Z}_{T}(\sigma, z)=$ $\widetilde{Z}(\sigma, z)-\widetilde{Z}_{N}(\sigma, z) n(\sigma)$ is its tangential part, and where :

$$
G_{s}(\sigma)=\frac{1}{\gamma_{s}(\sigma)} \frac{\partial \gamma_{s}}{\partial s}(\sigma)
$$

Laplace operator : for $\tilde{v}: \Gamma \longrightarrow \mathbb{R}$ we define

$$
\Delta_{\Gamma_{s}} \tilde{v}=\operatorname{div}_{\Gamma_{s}} \nabla_{\Gamma_{s}} \tilde{v}
$$

and if $u: \omega_{\eta_{0}} \longrightarrow \mathbb{R}$, denoting $\tilde{u}=u \circ \Theta$, we have :

$$
\begin{aligned}
\Delta u(x)= & \frac{\partial^{2} \tilde{u}}{\partial z^{2}}(P(x), \varphi(x))+G_{\varphi(x)}(P(x)) \frac{\partial \tilde{u}}{\partial z}(P(x), \varphi(x)) \\
& +\left(\Delta_{\Gamma_{\varphi(x)}} \tilde{u}\right)(P(x), \varphi(x)) .
\end{aligned}
$$

Remark 2.1 All these expressions are proved in detail in [9].

\subsection{Analytical tools}

We recall the following lemma, proved in [12] (see Theorem 2.1 on page 18), concerning the relevement of the divergence in a fixed domain:

Proposition 2.1 There exists a constant $C$ such that for all $g \in L^{2}(\Omega)$ such that $\int_{\Omega} g=0$, there exists $\psi \in H_{0}^{1}(\Omega)$ with $\operatorname{div} \psi=g$ in $\Omega$ and

$$
\|\psi\|_{H_{0}^{1}(\Omega)} \leq C\|g\|_{L^{2}(\Omega)}
$$

For the domain $\omega_{\varepsilon}$, depending on $\varepsilon$, we prove in [9] the same kind of result:

Proposition 2.2 There exists a constant $C$ such that for $\varepsilon$ small enough, for all $g \in L^{2}\left(\omega_{\varepsilon}\right)$ satisfying $\int_{\omega_{\varepsilon}} g=0$, there exists $\psi_{\varepsilon} \in\left(H_{0}^{1}\left(\omega_{\varepsilon}\right)\right)^{3}$ such that :

$$
\left\{\begin{array}{l}
\operatorname{div} \psi_{\varepsilon}=g \in \omega_{\varepsilon}, \\
\left\|\psi_{\varepsilon}\right\|_{H^{1}\left(\omega_{\varepsilon}\right)} \leq \frac{C}{\varepsilon}\|g\|_{L^{2}\left(\omega_{\varepsilon}\right)} .
\end{array}\right.
$$

We mention now a result concerning the harmonic extension of a boundary value in a variable domain depending on $\varepsilon$.

Proposition 2.3 Let $\varepsilon<\eta_{0}$, let $g \in H^{\frac{3}{2}}(\Gamma)$. Let us consider $r_{\varepsilon}$ the harmonic extension of $g \circ P$ in $\Omega_{\varepsilon}$, that is $r_{\varepsilon}$ is defined by

$$
\left\{\begin{array}{l}
\Delta r_{\varepsilon}=0 \text { in } \Omega_{\varepsilon}, \\
r_{\varepsilon}(x)=g(P(x)) \text { on } \Gamma_{\varepsilon},
\end{array}\right.
$$


Then there exists a constant $C$ independant of $\varepsilon$ and $g$ such that

$$
\left\|r_{\varepsilon}\right\|_{H^{2}\left(\Omega_{\varepsilon}\right)} \leq C\|g\|_{H^{\frac{3}{2}}(\Gamma)} .
$$

This proposition is a straightforward adaptation of the same result for a fixed regular domain (see [1] for the existence of a $H^{2}$ extension of the boundary value, and [11] for the resolution of the Laplace equation in $\Omega_{\varepsilon}$ ).

\section{Brinkmann Model}

\subsection{Formal asymptotic expansion}

We denote by $v^{\varepsilon}$ (resp. $q^{\varepsilon}$ ) the restriction of $u^{\varepsilon}$ (resp. $p^{\varepsilon}$ ) in $\omega_{\varepsilon}$. We write Equation (1.2) on the form:

$$
\left\{\begin{array}{lc}
\frac{\partial u^{\varepsilon}}{\partial t}-\Delta u^{\varepsilon}+\left(u^{\varepsilon} \cdot \nabla\right) u^{\varepsilon}+\nabla p^{\varepsilon}=f & \text { in } \mathbb{R}_{t}^{+} \times \Omega_{e x t} \\
\operatorname{div} u^{\varepsilon}=0 & \text { in } \mathbb{R}_{t}^{+} \times \Omega_{\text {ext }} \\
-\Delta v^{\varepsilon}+\frac{1}{\varepsilon} v^{\varepsilon}+\nabla q^{\varepsilon}=0 & \text { in } \mathbb{R}_{t}^{+} \times \omega_{\varepsilon} \\
\operatorname{div} v^{\varepsilon}=0 & \text { in } \mathbb{R}_{t}^{+} \times \omega_{\varepsilon} \\
u^{\varepsilon}=v^{\varepsilon} & \text { on } \mathbb{R}_{t}^{+} \times \partial \omega \\
-\frac{\partial u^{\varepsilon}}{\partial n}+p^{\varepsilon} n=-\frac{\partial v^{\varepsilon}}{\partial n}+q^{\varepsilon} n+\frac{1}{2}\left|u^{\varepsilon}\right|^{2} n & \text { on } \mathbb{R}_{t}^{+} \times \partial \omega \\
v^{\varepsilon}=0 & \text { in } \mathbb{R}_{t}^{+} \times \Gamma_{\varepsilon} \\
u^{\varepsilon}=0 & \text { on } \mathbb{R}_{t}^{+} \times \partial \mathcal{O} \\
u^{\varepsilon}(t=0)=u_{0} & \text { on } \Omega_{e x t} \\
v^{\varepsilon}(t=0)=0 & \text { on } \omega_{\varepsilon}
\end{array}\right.
$$

On one hand we assume that $u^{\varepsilon}$ and $p^{\varepsilon}$ admit the following asymptotic expansion:

$$
\begin{aligned}
& u^{\varepsilon}(t, x)=U^{0}(t, x)+\varepsilon U^{1}(t, x)+\ldots, \\
& p^{\varepsilon}(t, x)=p^{0}(t, x)+\varepsilon p^{1}(t, x)+\ldots
\end{aligned}
$$

where the profiles $U^{i}$ and $p^{i}$ are defined for $(t, x) \in \mathbb{R}^{+} \times \Omega_{e x t}$.

On the other hand we suppose that $v^{\varepsilon}$ and $q^{\varepsilon}$ admit an asympotitc expansion of the form: 


$$
\begin{aligned}
& v^{\varepsilon}(t, x)=V^{0}\left(t, P(x), \frac{\varphi(x)}{\varepsilon}\right)+\varepsilon V^{1}\left(t, P(x), \frac{\varphi(x)}{\varepsilon}\right)+\ldots, \\
& q^{\varepsilon}(t, x)=q^{0}\left(t, P(x), \frac{\varphi(x)}{\varepsilon}\right)+\varepsilon q^{1}\left(t, P(x), \frac{\varphi(x)}{\varepsilon}\right)+\varepsilon^{2} q^{2}\left(t, P(x), \frac{\varphi(x)}{\varepsilon}\right)+\ldots
\end{aligned}
$$

where the profiles $V^{i}$ and $q^{i}$ are defined on $\mathbb{R}_{t}^{+} \times \Gamma \times[0, \kappa]$.

In order to satisfy Equation (3.1.7) we suppose that:

$$
\forall t \in \mathbb{R}^{+}, \forall \sigma \in \Gamma, \quad V^{i}(t, \sigma, z=\kappa)=0 .
$$

Using the notations of Section 2.1 we recall that if $\tilde{\alpha}: \Gamma \times[0, \kappa] \longrightarrow \mathbb{R}$, if we denote $\alpha(x)=\tilde{\alpha}\left(P(x), \frac{\varphi(x)}{\varepsilon}\right)$, then

$$
\nabla \alpha(x)=\frac{1}{\varepsilon} \frac{\partial \tilde{\alpha}}{\partial z}\left(P(x), \frac{\varphi(x)}{\varepsilon}\right) n(P(x))+\nabla_{\Gamma_{\varphi(x)}} \tilde{\alpha}\left(P(x), \frac{\varphi(x)}{\varepsilon}\right) .
$$

Furthermore, if $\tilde{\beta}: \Gamma \times[0, \kappa] \longrightarrow \mathbb{R}^{3}$, if $\beta(x)=\tilde{\beta}\left(P(x), \frac{\varphi(x)}{\varepsilon}\right)$, then

$$
\begin{aligned}
\operatorname{div} \beta(x)= & \frac{1}{\varepsilon} \frac{\partial \tilde{\beta}_{N}}{\partial z}\left(P(x), \frac{\varphi(x)}{\varepsilon}\right)+G_{\varphi(x)}(P(x)) \tilde{\beta}_{N}\left(P(x), \frac{\varphi(x)}{\varepsilon}\right) \\
& +\operatorname{div} \Gamma_{\varphi(x)} \tilde{\beta}_{T}\left(P(x), \frac{\varphi(x)}{\varepsilon}\right) .
\end{aligned}
$$

In addition, if $\tilde{\alpha}: \Gamma \times[0, \kappa] \longrightarrow \mathbb{R}$, if we denote $\alpha(x)=\tilde{\alpha}\left(P(x), \frac{\varphi(x)}{\varepsilon}\right)$, then

$$
\begin{aligned}
\Delta \alpha(x)= & \frac{1}{\varepsilon^{2}} \frac{\partial^{2} \tilde{\alpha}}{\partial z^{2}}\left(P(x), \frac{\varphi(x)}{\varepsilon}\right)+\frac{1}{\varepsilon} G_{\varphi(x)}(P(x)) \frac{\partial \tilde{\alpha}}{\partial z}\left(P(x), \frac{\varphi(x)}{\varepsilon}\right) \\
& +\Delta_{\Gamma_{\varphi(x)}} \tilde{\alpha}\left(P(x), \frac{\varphi(x)}{\varepsilon}\right) .
\end{aligned}
$$

We plug the formal asymptotic expansions of $u^{\varepsilon}, p^{\varepsilon}, v^{\varepsilon}$ and $q^{\varepsilon}$ in System (3.1) and we identify the different powers of $\varepsilon$. Using this BKW method we can identify the different profiles in the asymptotic expansions.

\subsubsection{Determination of $U^{0}$ and $V^{0}$}

Writing (3.1.3) at order $\varepsilon^{-2}$ we obtain that $V_{z z}^{0}=0$. With (3.1.6) at order $\varepsilon^{-1}$, we obtain that $V_{z}^{0}=0$ for $z=0$. Thus, since $V^{0}(z=\kappa)=0$ we have

$$
V^{0} \equiv 0 .
$$


Therefore, with (3.1.5) at order $\varepsilon^{0}$, we know that $U^{0}=0$ on $\Gamma$. Writing (3.1.1), (3.1.2), (3.1.8) and (3.1.9) at order $\varepsilon^{0}$ we characterize $U^{0}$ by

$$
\left\{\begin{array}{l}
\frac{\partial U^{0}}{\partial t}-\Delta U^{0}+\left(U^{0} \cdot \nabla\right) U^{0}+\nabla p^{0}=f \text { in } \Omega_{e x t}, \\
\operatorname{div} U^{0}=0 \text { in } \Omega_{e x t} \\
U^{0}=0 \text { on } \Gamma \cup \partial \mathcal{O} . \\
U^{0}(t=0)=u_{0}
\end{array}\right.
$$

that is $\left(U^{0}, p^{0}\right)$ is our regular solutino of (1.3)-(1.4).

\subsubsection{Order $\varepsilon^{1}$ terms}

With (3.1.4) at order $\varepsilon^{0}$, and with (3.1.7) we obtain that

$$
V_{N}^{1} \equiv 0 \text {. }
$$

Now we write (3.1.3) at order $\varepsilon^{-1}$. We obtain that

$$
-\frac{\partial^{2} V^{1}}{\partial z^{2}}+\frac{\partial q^{0}}{\partial z} n=0
$$

Taking the normal part of (3.5) we obtain that

$$
\frac{\partial q^{0}}{\partial z}=0
$$

and with the tangential part of (3.5) we obtain that $V_{T}^{1}$ is an affine map with respect to the variable $z$.

Equation (3.1.6) at order $\varepsilon^{0}$ gives that

$$
-\frac{\partial U^{0}}{\partial n}+p^{0} n=-\frac{\partial V^{1}}{\partial z}+q^{0} n \text { for } z=0 .
$$

Taking the normal part, since $\frac{\partial U^{0}}{\partial n}$ is tangential at the boundary, since $V^{1}$ is tangential, we obtain that $p^{0}=q^{0}$ on $\Gamma$, and with (3.6) we obtain that

$$
q^{0}(t, \sigma, z)=p^{0}(t, \sigma) .
$$

With the tangential part of $(3.7)$, since $V_{T}^{1}(z=\kappa)=0$ and since $V_{T}^{1}$ is affine, we obtain that

$$
V_{T}^{1}(t, \sigma, z)=(z-\kappa) \frac{\partial U^{0}}{\partial n}(t, \sigma) .
$$


We can then determine $U^{1}$ with (3.1.1), (3.1.2), (3.1.5), (3.1.8) and (3.1.9) at order $\varepsilon^{1}$ :

$$
\left\{\begin{array}{l}
\frac{\partial U^{1}}{\partial t}-\Delta U^{1}+\left(U^{0} \cdot \nabla\right) U^{1}+\left(U^{1} \cdot \nabla\right) U^{0}+\nabla p^{1}=0 \text { in } \Omega_{e x t} \\
\operatorname{div} U^{1}=0 \text { in } \Omega_{e x t} \\
U^{1}=-\kappa \frac{\partial U^{0}}{\partial n} \text { on } \Gamma \\
U^{1}=0 \text { on } \partial \mathcal{O} \\
U^{1}(t=0)=0 \text { on } \Omega_{e x t}
\end{array}\right.
$$

\subsubsection{Determination of the order $\varepsilon^{2}$ terms}

Writing (3.1.4) at order $\varepsilon^{1}$ we obtain that $\frac{\partial V_{N}^{2}}{\partial z}+\operatorname{div}_{\Gamma} V_{T}^{1}=0$ and since $V^{2}(z=\kappa)=0$ we have:

$$
V_{N}^{2}(t, \sigma, z)=-\frac{1}{2}(z-\kappa)^{2} \operatorname{div}_{\Gamma}\left(\frac{\partial U^{0}}{\partial n}{ }_{\mid \Gamma}\right)(t, \sigma) .
$$

With (3.1.3) at order $\varepsilon^{0}$ we have

$$
-G_{0} \frac{\partial V^{1}}{\partial z}-\frac{\partial^{2} V^{2}}{\partial z^{2}}+V^{1}+\frac{\partial q^{1}}{\partial z} n+\nabla_{\Gamma} q^{0}=0
$$

With (3.1.6) at order $\varepsilon^{1}$ we obtain

$$
-\frac{\partial U^{1}}{\partial n}+p^{1} n=-\frac{\partial V^{2}}{\partial z}+q^{1} n \text { for } z=0 .
$$

Taking the normal parts of (3.12) and (3.13) we obtain that

$$
q^{1}(t, \sigma, z)=p^{1}(t, \sigma)-\left(\frac{\partial U^{1}}{\partial n}\right)_{N}(t, \sigma)+(\kappa-z) \operatorname{div} \Gamma_{\Gamma}\left(\frac{\partial U^{0}}{\partial n} \mid \Gamma\right)(t, \sigma) .
$$

Taking the tangential part of (3.12) and (3.13) we characterize $V_{T}^{2}$ by:

$$
\begin{aligned}
V_{T}^{2}(t, \sigma, z) & =\frac{1}{6}(z-\kappa)^{3} \frac{\partial U^{0}}{\partial n}(t, \sigma)+\frac{1}{2}(z-\kappa)^{2}\left(-G_{0} \frac{\partial U^{0}}{\partial n}+\nabla p^{0}\right)(t, \sigma) \\
& +(z-\kappa)\left(\left(\frac{\partial U^{1}}{\partial n}\right)_{T}-\frac{\kappa^{2}}{2} \frac{\partial U^{0}}{\partial n}+\kappa\left(-G_{0} \frac{\partial U^{0}}{\partial n}+\nabla p^{0}\right)\right)(t, \sigma) .
\end{aligned}
$$


Taking the value of $V^{2}$ for $z=0$, taking (3.1.2) and (3.1.5) at order $\varepsilon^{2}$ we prescribe $U^{2}$ by:

$$
\left\{\begin{array}{l}
\operatorname{div} U^{2}=0 \text { in } \Omega_{e x t}, \\
U^{2}=0 \text { on } \partial \mathcal{O}, \\
U^{2}(t, \sigma)=V^{2}(t, \sigma) \text { for } \sigma \in \Gamma .
\end{array}\right.
$$

\subsection{Existence and regularity for the profiles}

In this subsection we prove the existence of regular profiles satisfying the equations found by the BKW method.

We recall that $\left(U^{0}, p^{0}\right)$ is a regular solution of the Navier Stokes system (1.3) defined in $\left[0, T^{\star}\left[\times \Omega_{\text {ext }}\right.\right.$, with initial data $U^{0}(t=0)=u_{0}$.

The existence of the profile $U^{1}$ is claimed in the following

Proposition 3.1 There exists $\left(U^{1}, p^{1}\right)$ solution of Equation (3.10) on $\left[0, T^{*}\left[\times \Omega_{\text {ext }}\right.\right.$ and satisfying that for all $T<T^{*}$,

$$
\left\{\begin{array}{l}
\frac{\partial^{i} U^{1}}{\partial t^{i}} \in L^{\infty}\left(0, T ; H^{4-2 i}\left(\Omega_{e x t}\right)\right) \cap L^{2}\left(0, T ; H^{5-2 i}\left(\Omega_{e x t}\right)\right) \text { for } i=0,1,2, \\
\frac{\partial^{i} p^{1}}{\partial t^{i}} \in L^{\infty}\left(0, T ; H^{3-2 i}\left(\Omega_{e x t}\right)\right) \cap L^{2}\left(0, T ; H^{4-2 i}\left(\Omega_{e x t}\right)\right) \text { for } i=0,1 .
\end{array}\right.
$$

Sketch of the proof: we consider a relevement $\Upsilon^{1}$ of the boundary condition, which satisfies :

$$
\left\{\begin{array}{l}
\frac{\partial^{i} \Upsilon}{\partial t^{i}} \in L^{\infty}\left(0, T ; H^{3-2 i}\left(\Omega_{e x t}\right)\right) \cap L^{2}\left(0, T ; H^{4-2 i}\left(\Omega_{e x t}\right)\right) \text { for } i=0,1 \\
\operatorname{div} \Upsilon^{1}=0 \\
A \Upsilon^{1}=0 \\
\Upsilon^{1}=0 \text { on } \partial \mathcal{O} \\
\Upsilon^{1}=\frac{\partial U^{0}}{\partial n} \text { on } \Gamma .
\end{array}\right.
$$

Writting $U^{1}=Z^{1}+\Upsilon^{1}$, we are led to prove the existence of a sufficiently regular 
solution for the following equation:

$$
\left\{\begin{array}{l}
\frac{\partial Z^{1}}{\partial t}-\Delta Z^{1}+\left(U^{0} \cdot \nabla\right) Z^{1}+\left(Z^{1} \cdot \nabla\right) U^{0}+\nabla q^{1}=Q^{1} \\
\operatorname{div} Z^{1}=0 \\
Z^{1}=0 \text { on } \partial \Omega_{e x t},
\end{array}\right.
$$

where $Q^{1}=-\frac{\partial \Upsilon^{1}}{\partial t}-\left(U^{0} \cdot \nabla\right) \Upsilon^{1}-\left(\Upsilon^{1} \cdot \nabla\right) U^{0}$.

We build a regular solution $Z^{1}$ as we build a regular solution for Navier Stokes equation (see [4] or [10]). In particular we obtain more regularity derivating the Galerkin approximation of Equation (3.18) with respect to $t$.

We can deduce from Proposition 3.1 the regularity for the other profiles:

Proposition 3.2 Under condition (1.4) on $U^{0}$ and under hypothesis of Proposition 3.1, we have the following regularity results: for $T<T^{\star}$,

- $V^{1}$ defined by (3.4) and (3.9) satisfies:

$$
\frac{\partial^{i} V^{1}}{\partial t^{i}} \in L^{\infty}\left(0, T ; H^{\frac{7}{2}-2 i}(\Gamma) \otimes \mathcal{C}^{\infty}(0, \kappa)\right) \cap L^{2}\left(0, T ; H^{\frac{9}{2}-2 i}(\Gamma) \otimes \mathcal{C}^{\infty}(0, \kappa)\right),
$$

- $q^{0}$ defined by (3.8) satisfies:

$$
\frac{\partial^{i} q^{0}}{\partial t^{i}} \in L^{\infty}\left(0, T ; H^{\frac{7}{2}-2 i}(\Gamma) \otimes \mathcal{C}^{\infty}(0, \kappa)\right) \cap L^{2}\left(0, T ; H^{\frac{9}{2}-2 i}(\Gamma) \otimes \mathcal{C}^{\infty}(0, \kappa)\right),
$$

- $V^{2}$ defined by (3.11) and (3.15) satisfies:

$$
\frac{\partial^{i} V^{2}}{\partial t^{i}} \in L^{\infty}\left(0, T ; H^{\frac{5}{2}-2 i}(\Gamma) \otimes \mathcal{C}^{\infty}(0, \kappa)\right) \cap L^{2}\left(0, T ; H^{\frac{7}{2}-2 i}(\Gamma) \otimes \mathcal{C}^{\infty}(0, \kappa)\right),
$$

- $q^{1}$ defined by (3.14) satisfies:

$$
\frac{\partial^{i} q^{1}}{\partial t^{i}} \in L^{\infty}\left(0, T ; H^{\frac{5}{2}-2 i}(\Gamma) \otimes \mathcal{C}^{\infty}(0, \kappa)\right) \cap L^{2}\left(0, T ; H^{\frac{7}{2}-2 i}(\Gamma) \otimes \mathcal{C}^{\infty}(0, \kappa)\right),
$$

- $U^{2}$ defined by (3.16) satisfies:

$$
\frac{\partial^{i} U^{2}}{\partial t^{i}} \in L^{\infty}\left(0, T ; H^{3-2 i}\left(\Omega_{e x t}\right)\right) \cap L^{2}\left(0, T ; H^{4-2 i}\left(\Omega_{e x t}\right)\right) .
$$




\subsection{Estimate of the remainder term for Theorem 1.1}

We define $U_{a}$ (resp. $V_{a}$ ) and $p_{a}$ (resp. $q_{a}$ ) the approximations of $u^{\varepsilon}$ and $p^{\varepsilon}$ in $\Omega_{\text {ext }}\left(\right.$ resp. $\left.\omega_{\varepsilon}\right)$ given by

$$
\left\{\begin{array}{l}
U_{a}(t, x)=U^{0}(t, x)+\varepsilon U^{1}(t, x)+\varepsilon^{2} U^{2}(t, x) \text { for }(t, x) \in\left[0, T^{\star}\left[\times \Omega_{e x t},\right.\right. \\
V_{a}(t, x)=\varepsilon V^{1}\left(t, P(x), \frac{\varphi(x)}{\varepsilon}\right)+\varepsilon^{2} V^{2}\left(t, P(x), \frac{\varphi(x)}{\varepsilon}\right) \text { for }(t, x) \in\left[0, T^{\star}\left[\times \omega_{e},\right.\right. \\
p_{a}(t, x)=p^{0}(t, x)+\varepsilon p^{1}(t, x) \text { for }(t, x) \in\left[0, T^{\star}\left[\times \Omega_{e x t},\right.\right. \\
q_{a}(t, x)=q^{0}\left(t, P(x), \frac{\varphi(x)}{\varepsilon}\right)+\varepsilon q^{1}\left(t, P(x), \frac{\varphi(x)}{\varepsilon}\right) \text { for }(t, x) \in\left[0, T^{\star}\left[\times \omega_{\varepsilon} .\right.\right.
\end{array}\right.
$$

Applying Proposition 2.2, we consider $\Psi^{\varepsilon} \in L^{\infty}\left(0, T ; H_{0}^{1}\left(\omega_{\varepsilon}\right)\right)$ satisfying

$$
\operatorname{div} \Psi^{\varepsilon}=-\frac{1}{\varepsilon^{\frac{3}{2}}} \operatorname{div} V_{a} .
$$

We remark that

$$
\operatorname{div} V_{a}=\varepsilon\left(\operatorname{div}_{\Gamma_{\varphi(x)}} V_{T}^{1}-\operatorname{div}_{\Gamma_{0}} V_{T}^{1}\right)+\varepsilon^{2} \operatorname{div}_{\Gamma_{\varphi(x)}} V_{T}^{2} .
$$

With the estimates performed on the different terms of the anzatz we remark that

$$
\left\|\frac{1}{\varepsilon^{\frac{3}{2}}} \operatorname{div} V_{a}\right\|_{L^{2}\left(\omega_{\varepsilon}\right)} \leq C \varepsilon,
$$

and then, with Proposition 2.2, we can assume that

$$
\left\|\Psi^{\varepsilon}\right\|_{H^{1}\left(\omega_{\varepsilon}\right)} \leq C .
$$

and with the Poincaré inequality in the domain $\omega_{\varepsilon}$, we have

$$
\left\|\Psi^{\varepsilon}\right\|_{L^{2}\left(\omega_{\varepsilon}\right)} \leq C \varepsilon
$$

We define now the remainder term $r^{\varepsilon}$ by :

$$
u^{\varepsilon}(t, x)=\left\{\begin{array}{l}
U_{a}(t, x)+\varepsilon^{\frac{3}{2}} r^{\varepsilon}(t, x) \text { for } x \in \Omega_{e x t}, \\
V_{a}(t, x)+\varepsilon^{\frac{3}{2}} \psi^{\varepsilon}(t, x)+\varepsilon^{\frac{3}{2}} r^{\varepsilon}(t, x) \text { for } x \in \omega_{\varepsilon} .
\end{array}\right.
$$

We remark that $r^{\varepsilon} \in H_{0}^{1}\left(\Omega_{f l u}^{\varepsilon}\right)$ and $\operatorname{div} r^{\varepsilon}=0$ on $\Omega_{f l u}^{\varepsilon}$. We take $r^{\varepsilon}$ as a test function in the weak formulation of (1.2). Dividing by $\varepsilon^{\frac{3}{2}}$ we obtain that :

$$
\frac{1}{2} \frac{d}{d t}\left\|r^{\varepsilon}\right\|_{L^{2}\left(\Omega_{e x t}\right)}^{2}+\left\|\nabla r^{\varepsilon}\right\|_{L^{2}\left(\Omega_{f l u}^{\varepsilon}\right)}^{2}+\frac{1}{\varepsilon} \int_{\omega_{\varepsilon}}\left|r^{\varepsilon}\right|^{2}=T_{1}+\ldots+T_{5},
$$


with

$$
\begin{aligned}
T_{1}= & -\varepsilon^{\frac{3}{2}} \int_{\Omega_{e x t}}\left(r^{\varepsilon} \cdot \nabla\right) r^{\varepsilon} \cdot r^{\varepsilon}+\frac{1}{2} \varepsilon^{\frac{3}{2}} \int_{\Gamma}\left|r^{\varepsilon}\right|^{2}\left(r^{\varepsilon} \cdot n\right), \\
T_{2}= & \frac{1}{\varepsilon^{\frac{3}{2}}}\left[-\int_{\Omega_{e x t}} \frac{\partial U_{a}}{\partial t} r^{\varepsilon}-\int_{\Omega_{e x t}} \nabla U_{a} \cdot \nabla r^{\varepsilon}-\int_{\Omega_{e x t}}\left(U_{a} \cdot \nabla\right) U_{a} \cdot r^{\varepsilon}\right] \\
& +\frac{1}{\varepsilon^{\frac{3}{2}}}\left[+\int_{\Omega_{e x t}} f r^{\varepsilon}-\int_{\omega_{\varepsilon}} \nabla V_{a} \cdot \nabla r^{\varepsilon}-\frac{1}{\varepsilon} \int_{\omega_{\varepsilon}} V_{a} r^{\varepsilon}\right], \\
T_{3}= & -\int_{\omega_{\varepsilon}} \nabla \Psi_{\varepsilon} \nabla r^{\varepsilon}-\frac{1}{\varepsilon} \int_{\omega_{\varepsilon}} \Psi_{\varepsilon} r^{\varepsilon}, \\
T_{4}= & -\int_{\Omega_{e x t}}\left(r^{\varepsilon} \cdot \nabla\right) U_{a} \cdot r^{\varepsilon}-\int_{\Omega_{e x t}}\left(U_{a} \cdot \nabla\right) r^{\varepsilon} \cdot r^{\varepsilon}, \\
T_{5}= & -\frac{1}{2 \varepsilon^{\frac{3}{2}}} \int_{\Gamma}\left|U_{a}\right|^{2} r^{\varepsilon} \cdot n-\int_{\Gamma}\left(U_{a} \cdot r^{\varepsilon}\right) r^{\varepsilon} \cdot n .
\end{aligned}
$$

We remark first that $T_{1}=0$. Indeed,

$$
\begin{aligned}
\int_{\Omega_{e x t}}\left(r^{\varepsilon} \cdot \nabla\right) r^{\varepsilon} \cdot r^{\varepsilon} & =\int_{\Omega_{e x t}} \sum_{i, j} r_{i}^{\varepsilon} \frac{\partial r_{j}^{\varepsilon}}{\partial x_{i}} r_{j}^{\varepsilon} \\
& =\frac{1}{2} \sum_{i, j} \int_{\Omega_{e x t}} r_{i}^{\varepsilon} \frac{\partial}{\partial x_{i}}\left(r_{j}^{\varepsilon}\right)^{2} \\
& =-\frac{1}{2} \sum_{i, j} \int_{\Omega_{e x t}} \frac{\partial r_{i}^{\varepsilon}}{\partial x_{i}}\left(r_{j}^{\varepsilon}\right)^{2}+\frac{1}{2} \int_{\Gamma}\left|r^{\varepsilon}\right|^{2}\left(r^{\varepsilon} \cdot n\right) \\
& =\frac{1}{2} \int_{\Gamma}\left|r^{\varepsilon}\right|^{2}\left(r^{\varepsilon} \cdot n\right) .
\end{aligned}
$$

The estimates of the other terms are given by the following lemmas:

Lemma 3.1 For all $\eta>0$ there exists a constant $C(\eta)$ such that

$$
\left|T_{2}\right| \leq \eta\left\|\nabla r^{\varepsilon}\right\|_{L^{2}\left(\Omega_{f l u}^{\varepsilon}\right)}^{2}+\frac{\eta}{\varepsilon}\left\|r^{\varepsilon}\right\|_{L^{2}\left(\omega_{\varepsilon}\right)}^{2}+C(\eta)\left(1+\left\|r^{\varepsilon}\right\|_{L^{2}\left(\Omega_{e x t}\right)}^{2}\right) .
$$

Proof: we first remark that

$$
\int_{\Omega_{e x t}} \nabla U_{a} \nabla r^{\varepsilon}=\int_{\Omega_{e x t}}\left(\nabla U^{0}+\varepsilon \nabla U^{1}\right) \nabla r^{\varepsilon}-\int_{\Omega_{e x t}}\left(p^{0}+\varepsilon p^{1}\right) \operatorname{div} r^{\varepsilon}+\varepsilon^{2} \int_{\Omega_{e x t}} \nabla U^{2} \nabla r^{\varepsilon},
$$


since $\operatorname{div} r^{\varepsilon}=0$. We integrate by part the two firts integrals and we obtain that

$$
\begin{aligned}
\int_{\Omega_{e x t}} \nabla U_{a} \nabla r^{\varepsilon}= & -\int_{\Omega_{e x t}}\left[\left(\Delta U^{0}-\nabla p^{0}\right)+\varepsilon\left(\Delta U^{1}-\nabla p^{1}\right)\right] r^{\varepsilon}+\varepsilon^{2} \int_{\Omega_{e x t}} \nabla U^{2} \nabla r^{\varepsilon} \\
& +\int_{\Gamma}\left[\left(\frac{\partial U^{0}}{\partial n}-p^{0} n\right)+\varepsilon\left(\frac{\partial U^{1}}{\partial n}-p^{1} n\right)\right] r^{\varepsilon}
\end{aligned}
$$

In the same way,

$$
\int_{\omega_{\varepsilon}} \nabla V_{a} \nabla r^{\varepsilon}=-\int_{\omega_{\varepsilon}}\left(\Delta V_{a}-\nabla q_{a}\right) r^{\varepsilon}-\int_{\Gamma}\left[\varepsilon \frac{\partial V_{a}}{\partial n}-q_{a} n\right] r^{\varepsilon} .
$$

Thus, using the equations satisfied by the profiles, $T_{2}=T_{21}+T_{22}$ with

$$
\begin{aligned}
T_{21}= & -\varepsilon^{\frac{1}{2}}\left(\int_{\Omega_{e x t}} \frac{\partial U^{2}}{\partial t} r^{\varepsilon}+\int_{\Omega_{e x t}}\left[\left(U^{0} \cdot \nabla\right) U^{2}+\left(U^{1} \cdot \nabla\right) U^{1}+\left(U^{2} \cdot \nabla\right) U^{0}\right] r^{\varepsilon}\right) \\
& -\varepsilon^{\frac{3}{2}} \int_{\Omega_{e x t}}\left(\left(U^{1} \cdot \nabla\right) U^{2}+\left(U^{2} \cdot \nabla\right) U^{1}+\varepsilon\left(U^{2} \cdot \nabla\right) U^{2}\right) r^{\varepsilon} \\
& -\varepsilon^{\frac{1}{2}} \int_{\Omega_{e x t}} \nabla U^{2} \cdot \nabla r^{\varepsilon}, \\
T_{22}= & \frac{1}{\varepsilon^{\frac{1}{2}}} \int_{\omega_{\varepsilon}} K_{\varepsilon}\left(t, P(x), \frac{\varphi(x)}{\varepsilon}\right) r^{\varepsilon}(x),
\end{aligned}
$$

where

$$
\begin{aligned}
K_{\varepsilon}(t, \sigma, z)= & \frac{1}{\varepsilon}\left(G_{\varepsilon z}-G_{0}\right) \frac{\partial V^{1}}{\partial z}+\Delta_{\Gamma_{\varepsilon z}} V^{1}+G_{\varepsilon z} \frac{\partial V^{2}}{\partial z}+\varepsilon \Delta_{\Gamma_{\varepsilon z}} V^{2}-V^{2} \\
& +\frac{1}{\varepsilon}\left(\nabla_{\Gamma_{\varepsilon z}}-\nabla_{\Gamma}\right) q^{0}+\nabla_{\Gamma_{\varepsilon z}} q^{1} .
\end{aligned}
$$

With the estimates obtained for the profiles,

$$
\begin{aligned}
\left|T_{21}\right| \leq & \varepsilon^{\frac{1}{2}} C\left\|r^{\varepsilon}\right\|_{L^{2}\left(\Omega_{e x t}\right)}+\varepsilon^{\frac{1}{2}}\left\|\nabla r^{\varepsilon}\right\|_{L^{2}\left(\Omega_{e x t}\right)} \\
& \leq \eta\left\|\nabla r^{\varepsilon}\right\|_{L^{2}\left(\Omega_{e x t}\right)}^{2}+C(\eta)\left(1+\left\|v_{\varepsilon}\right\|_{L^{2}\left(\Omega_{e x t}\right)}^{2}\right) .
\end{aligned}
$$

Since $s \mapsto G_{s}$ and $s \mapsto \nabla_{\Gamma_{s}}$ are regular, since the profiles $q^{i}$ and $V^{i}$ are polynomial in the variable $z$ and are in $L^{\infty}\left(0, T ; H^{2}(\Gamma)\right)$ in the variable $\sigma \in \Gamma$, we have:

$$
\left\|(t, x) \mapsto K_{\varepsilon}\left(t, P(x), \frac{\varphi(x)}{\varepsilon}\right)\right\|_{L^{\infty}\left(0, T ; L^{2}\left(\omega_{\varepsilon}\right)\right)} \leq C \varepsilon^{\frac{1}{2}}
$$

thus there exists a constant $C$ such that $\left|T_{12}\right| \leq C\left\|r^{\varepsilon}\right\|_{L^{2}\left(\omega_{\varepsilon}\right)}$ and absorbing $\left\|r^{\varepsilon}\right\|_{L^{2}\left(\omega_{\varepsilon}\right)}$, if we fix $\eta>0$ there exists $C(\eta)$ such that

$$
\left|T_{22}\right| \leq \frac{\eta}{\varepsilon}\left\|r^{\varepsilon}\right\|_{L^{2}\left(\omega_{\varepsilon}\right)}^{2}+C(\eta) .
$$


Adding the two previous inequality we conclude the proof of Lemma 3.1.

Lemma 3.2 For $\eta>0$ there exists a constant $C(\eta)$ such that

$$
\left|T_{3}\right| \leq \frac{\eta}{\varepsilon}\left\|r^{\varepsilon}\right\|_{L^{2}\left(\omega_{\varepsilon}\right)}^{2}+\eta\left\|\nabla r^{\varepsilon}\right\|_{L^{2}\left(\omega_{\varepsilon}\right)}^{2}+C(\eta) .
$$

Proof: We remark that

$$
\begin{aligned}
\left|T_{3}\right| & \leq\left\|\nabla \Psi_{\varepsilon}\right\|_{L^{2}\left(\omega_{\varepsilon}\right)}\left\|\nabla r^{\varepsilon}\right\|_{L^{2}\left(\omega_{\varepsilon}\right)}+\frac{1}{\varepsilon}\left\|\Psi_{\varepsilon}\right\|_{L^{2}\left(\omega_{\varepsilon}\right)}\left\|r^{\varepsilon}\right\|_{L^{2}\left(\omega_{\varepsilon}\right)} \\
& \leq \frac{\eta}{\varepsilon}\left\|r^{\varepsilon}\right\|_{L^{2}\left(\omega_{\varepsilon}\right)}^{2}+\eta\left\|\nabla r^{\varepsilon}\right\|_{L^{2}\left(\omega_{\varepsilon}\right)}^{2}+C(\eta),
\end{aligned}
$$

with (3.19) and (3.20).

Lemma 3.3 For $\eta>0$ there exists a constant $C(\eta)$ such that

$$
\left|T_{4}\right| \leq \eta\left\|\nabla r^{\varepsilon}\right\|_{L^{2}\left(\Omega_{e x t}\right)}^{2}+C(\eta)\left\|r^{\varepsilon}\right\|_{L^{2}\left(\Omega_{e x t}\right)}^{2} .
$$

Proof: We estimate $T_{4}$ in the following way:

$$
\begin{aligned}
& \left|T_{4}\right| \leq\left\|\nabla U_{a}\right\|_{L^{6}\left(\Omega_{e x t}\right)}\left\|r^{\varepsilon}\right\|_{L^{3}\left(\Omega_{e x t}\right)}\left\|\nabla r^{\varepsilon}\right\|_{L^{2}\left(\Omega_{e x t}\right)} \\
& +\left\|U_{a}\right\|_{L^{6}\left(\Omega_{e x t}\right)}\left\|r^{\varepsilon}\right\|_{L^{3}\left(\Omega_{e x t}\right)}\left\|\nabla r^{\varepsilon}\right\|_{L^{2}\left(\Omega_{e x t}\right)} \\
& \leq C\left\|\nabla r^{\varepsilon}\right\|_{L^{2}\left(\Omega_{e x t}\right)}^{\frac{3}{2}}\left\|r^{\varepsilon}\right\|_{L^{2}\left(\Omega_{e x t}\right)}^{\frac{1}{2}}+C\left\|r^{\varepsilon}\right\|_{L^{2}\left(\Omega_{e x t}\right)}^{2} \\
& \leq \eta\left\|\nabla r^{\varepsilon}\right\|_{L^{2}\left(\Omega_{e x t}\right)}^{2}+C(\eta)\left\|r^{\varepsilon}\right\|_{L^{2}\left(\Omega_{e x t}\right)}^{2}
\end{aligned}
$$

using the Sobolev inequalities, the estimates performed on $U_{a}$ and using Young inequalities.

Lemma 3.4 There exists a constant $C$ such that for $\eta>0$ there exists $\varepsilon_{0}>0$ such that if $\varepsilon<\varepsilon_{0}$ then

$$
\left|T_{5}\right| \leq \eta\left\|\nabla r^{\varepsilon}\right\|_{L^{2}\left(\Omega_{e x t}\right)}^{2}+C\left(1+\left\|r^{\varepsilon}\right\|_{L^{2}\left(\Omega_{e x t}\right)}^{2}\right)
$$

Proof: We have

$$
\begin{aligned}
\left|T_{5}\right| & \leq C \frac{1}{\varepsilon^{\frac{3}{2}}}\left\|U_{a}\right\|_{L^{3}(\Gamma)}^{2}\left\|r^{\varepsilon}\right\|_{L^{3}(\Gamma)}+C\left\|U_{a}\right\|_{L^{3}(\Gamma)}\left\|r^{\varepsilon}\right\|_{L^{3}(\Gamma)}^{2} \\
& \leq C \varepsilon^{\frac{1}{2}}\left\|r^{\varepsilon}\right\|_{H^{1}\left(\Omega_{e x t}\right)}+C \varepsilon\left\|r^{\varepsilon}\right\|_{H^{1}\left(\Omega_{e x t}\right)}^{2}
\end{aligned}
$$

since $\left\|U_{a}\right\|_{L^{3}(\Gamma)} \leq C \varepsilon$ and since $\left\|r^{\varepsilon}\right\|_{L^{3}(\Gamma)} \leq C\left\|r^{\varepsilon}\right\|_{H^{\frac{1}{2}(\Gamma)}} \leq C\left\|r^{\varepsilon}\right\|_{H^{1}\left(\Omega_{e x t}\right)}$. 
We fix $\eta>0$, and for $\varepsilon$ small enough, we obtain that

$$
\left|T_{5}\right| \leq \eta\left\|\nabla r^{\varepsilon}\right\|_{L^{2}\left(\Omega_{e x t}\right)}^{2}+C\left(1+\left\|r^{\varepsilon}\right\|_{L^{2}\left(\Omega_{e x t}\right)}^{2}\right) .
$$

End of the proof of Theorem 1.1:

Adding the different estimates on $T_{2}, \ldots, T_{5}$, we obtain that there exists a constant $K(\eta)$ such that:

$$
\begin{aligned}
\frac{1}{2} \frac{d}{d t}\left\|r^{\varepsilon}\right\|_{L^{2}\left(\Omega_{e x t}\right)}^{2}+\quad\left\|\nabla r^{\varepsilon}\right\|_{L^{2}\left(\Omega_{f l u}^{\varepsilon}\right)}^{2}+\frac{1}{\varepsilon}\left\|r^{\varepsilon}\right\|_{L^{2}\left(\omega_{\varepsilon}\right)}^{2} \leq \frac{2 \eta}{\varepsilon}\left\|r^{\varepsilon}\right\|_{L^{2}\left(\omega_{\varepsilon}\right)}^{2} \\
+4 \eta\left\|\nabla r^{\varepsilon}\right\|_{L^{2}\left(\Omega_{f l u}^{\varepsilon}\right)}+K(\eta)\left(1+\left\|r^{\varepsilon}\right\|_{L^{2}\left(\Omega_{e x t}\right)}^{2}\right)
\end{aligned}
$$

Taking $\eta=\frac{1}{8}$ there exists a constant $K$ such that:

$$
\frac{d}{d t}\left\|r^{\varepsilon}\right\|_{L^{2}\left(\Omega_{e x t}\right)}^{2}+\left\|\nabla r^{\varepsilon}\right\|_{L^{2}\left(\Omega_{f l u}^{\varepsilon}\right)}^{2}+\frac{1}{\varepsilon}\left\|r^{\varepsilon}\right\|_{L^{2}\left(\omega_{\varepsilon}\right)}^{2} \leq C\left(1+\left\|r^{\varepsilon}\right\|_{L^{2}\left(\Omega_{e x t}\right)}^{2}\right)
$$

and we conclude the proof with the Gronwall Lemma.

\section{BKW Method for Equation (1.1)}

\subsection{Characterization of the profiles}

We denote by $v^{\varepsilon}$ (resp. $w^{\varepsilon}$ ) the restriction of $u^{\varepsilon}$ in $\omega_{\varepsilon}$ (resp. $\Omega_{\varepsilon}$ ). Furthermore the pressure $\pi^{\varepsilon}$ is denoted by :

$$
\pi^{\varepsilon}(t, x)=\left\{\begin{array}{l}
p^{\varepsilon}(t, x) \text { if } x \in \Omega_{e x t}, \\
q^{\varepsilon}(t, x) \text { if } x \in \omega_{\varepsilon}, \\
r^{\varepsilon}(t, x) \text { if } x \in \Omega_{\varepsilon} .
\end{array}\right.
$$

Equation (1.1) is equivalent to the following system : 


$$
\left\{\begin{array}{l}
\frac{\partial u^{\varepsilon}}{\partial t}-\Delta u^{\varepsilon}+\left(u^{\varepsilon} \cdot \nabla\right) u^{\varepsilon}+\nabla p^{\varepsilon}=f \text { in } \Omega_{e x t} \\
\operatorname{div} u^{\varepsilon}=0 \text { in } \Omega_{e x t} \\
\frac{\partial v^{\varepsilon}}{\partial t}-\Delta v^{\varepsilon}+\left(v^{\varepsilon} \cdot \nabla\right) v^{\varepsilon}+\nabla q^{\varepsilon}+\frac{1}{\varepsilon} v^{\varepsilon}=0 \text { in } \omega_{\varepsilon} \\
\operatorname{div} v^{\varepsilon}=0 \text { in } \omega_{\varepsilon} \\
\frac{\partial w^{\varepsilon}}{\partial t}-\Delta w^{\varepsilon}+\left(w^{\varepsilon} \cdot \nabla\right) w^{\varepsilon}+\nabla r^{\varepsilon}+\frac{1}{\varepsilon^{2}} w^{\varepsilon}=0 \text { in } \Omega_{\varepsilon} \\
\operatorname{div} w^{\varepsilon}=0 \text { in } \Omega_{\varepsilon} \\
u^{\varepsilon}=v^{\varepsilon} \text { on } \Gamma \\
v^{\varepsilon}=w^{\varepsilon} \text { on } \Gamma_{\varepsilon} \\
-\frac{\partial u^{\varepsilon}}{\partial n}+p^{\varepsilon} n=-\frac{\partial v^{\varepsilon}}{\partial n}+q^{\varepsilon} n \text { on } \Gamma \\
-\frac{\partial v^{\varepsilon}}{\partial n}+q^{\varepsilon} n=-\frac{\partial w^{\varepsilon}}{\partial n}+r^{\varepsilon} n \text { on } \Gamma_{\varepsilon} \\
u^{\varepsilon}(t=0)=u_{0} \text { in } \Omega_{e x t} \\
v^{\varepsilon}(t=0)=0 \text { in } \omega_{\varepsilon} \\
w^{\varepsilon}(t=0)=0 \text { in } \Omega_{\varepsilon}
\end{array}\right.
$$

For $x \in \Omega_{e x t}$ we perform an asymtotic expansion on the form :

$$
\begin{aligned}
& u^{\varepsilon}(t, x)=U^{0}(t, x)+\varepsilon \mathbf{U}^{1}(t, x)+\ldots \\
& p^{\varepsilon}(t, x)=p^{0}(t, x)+\varepsilon p^{1}(t, x)+\ldots
\end{aligned}
$$

For $x \in \omega_{\varepsilon}$ the asymptotic expansion will be described by

$$
\begin{aligned}
& v^{\varepsilon}(t, x)=\mathbf{V}^{0}\left(t, P(x), \frac{\varphi(x)}{\varepsilon}\right)+\varepsilon \mathbf{V}^{1}\left(t, P(x), \frac{\varphi(x)}{\varepsilon}\right)+\ldots \\
& q^{\varepsilon}(t, x)=q^{0}\left(t, P(x), \frac{\varphi(x)}{\varepsilon}\right)+\varepsilon q^{1}\left(t, P(x), \frac{\varphi(x)}{\varepsilon}\right)+\ldots
\end{aligned}
$$

where the different profiles are defined on $\mathbb{R}_{t}^{+} \times \Gamma \times[0, \kappa]$, and where $P(x)$ (resp. $\varphi(x)$ ) denote the orthogonal projection of $x$ onto $\Gamma$ (resp. the distance from $x$ to $\Gamma$ ). 
For $x \in \Omega_{\varepsilon}$ we write

$$
\begin{aligned}
& w^{\varepsilon}(t, x)=\mathbf{W}^{0}\left(t, x, \frac{\psi(x)}{\varepsilon}\right)+\varepsilon \mathbf{W}^{1}\left(t, x, \frac{\psi(x)}{\varepsilon}\right)+\ldots \\
& r^{\varepsilon}(t, x)=r^{0}\left(t, x, \frac{\psi(x)}{\varepsilon}\right)+\varepsilon r^{1}\left(t, x, \frac{\psi(x)}{\varepsilon}\right)+\ldots
\end{aligned}
$$

where $\psi(x)$ is the distance from $x$ to $\Gamma_{\varepsilon}$.

Here the profiles are defined on $\mathbb{R}_{t}^{+} \times \Omega_{\varepsilon} \times \mathbb{R}_{\xi}^{+}$and can be splited in two terms:

$$
\mathbf{W}^{i}(t, x, \xi)=\overline{\mathbf{W}^{i}}(t, x)+\widetilde{\mathbf{W}^{i}}(t, x, \xi)
$$

where we suppose that $\widetilde{\mathbf{W}^{i}}$ and all its derivatives with respect to $x$ and $\xi$ tend to zero when $\xi$ tends to $+\infty$. The boundary layer is described by this term $\widetilde{\mathbf{W}^{i}}$.

\section{Determination of order 0 terms}

At order $\varepsilon^{-2}$ in (4.1.5) we obtain that $-\frac{\partial^{2} \mathbf{W}^{0}}{\partial \xi^{2}}+\mathbf{W}^{0}=0$. Taking the limit when $\xi$ tends to $+\infty$ we obtain that

$$
\overline{\mathbf{W}^{0}}=0,
$$

and that there exists a function $K^{0}: \Omega_{\varepsilon} \longrightarrow \mathbb{R}^{3}$ such that :

$$
\widetilde{\mathbf{W}^{0}}(x, \xi)=K^{0}(x) e^{-\xi} \text {. }
$$

At order $\varepsilon^{-2}$ in (4.1.3) we obtain that

$$
-\frac{\partial^{2} \mathbf{V}^{0}}{\partial z^{2}}=0 \text { in } \Gamma \times[0, \kappa]
$$

With (4.1.9) at order $\varepsilon^{-1}$ we have that $-\frac{\partial \mathbf{V}^{0}}{\partial z}=0$ for $z=0$ and thus $\mathbf{V}^{0}$ does not depend on $z$.

Now with (4.1.10) at order $\varepsilon^{-1}$ we have $\frac{\partial \mathbf{W}^{0}}{\partial \xi}(\xi=0)=-\frac{\partial \mathbf{V}^{0}}{\partial z}(z=\kappa)=0$. With (4.2) we obtain that

$$
\mathbf{W}^{0} \equiv 0 \text {. }
$$

Writing (4.1.8) at order 0 we obtain that $\mathbf{V}^{0}=0$ for $z=\kappa$ and since $\mathbf{V}^{0}$ does not depend on $z$ we have

$$
\mathbf{V}^{0} \equiv 0
$$

We are now able to caracterize $U^{0}$ with $(4.1 .1),(4.1 .2),(4.1 .7)$ and (4.1.11) at order 0 , and with $(4.5)$, we see that $\left(U^{0}, p^{0}\right)$ is our regular solution of Navier Stokes Equation (1.3) in $\Omega_{e x t}$. 


\section{Determination of order 1.}

Writing (4.1.6) at order $\varepsilon^{0}$ we obtain that $\frac{\partial \widetilde{\mathbf{W}_{N}^{1}}}{\partial \xi}=0$ and since $\widetilde{\mathbf{W}_{N}^{1}}$ tends to zero when $\xi$ tends to $+\infty$ we have :

$$
\widetilde{\mathbf{W}_{N}^{1}} \equiv 0
$$

With (4.1.5) at order $\varepsilon^{-1}$ we get

$$
-\frac{\partial^{2} \mathbf{W}^{1}}{\partial \xi^{2}}+\frac{\partial r^{0}}{\partial \xi} n+\mathbf{W}^{1}=0
$$

Performing the limit when $\xi \longrightarrow+\infty$ we have :

$$
\overline{\mathbf{W}^{1}} \equiv 0 \text {. }
$$

With (4.6) the normal part of (4.8) gives us that $\frac{\partial r^{0}}{\partial \xi}=0$ that is

$$
\widetilde{r^{0}} \equiv 0
$$

and there exists $K^{1}: \Omega_{\varepsilon} \longrightarrow \mathbb{R}^{3}$, a tangential vector field, such that

$$
\widetilde{\mathbf{W}^{1}}(x, \xi)=K^{1}(x) e^{-\xi} \text {. }
$$

Writing (4.1.4) at order 0 we obtain that $\frac{\partial \mathbf{V}_{N}^{1}}{\partial z}=0$. With (4.1.9) at order $\varepsilon^{1}$,

$$
\mathbf{V}_{N}^{1}(z=\kappa)=\mathbf{W}_{N}^{1}(\xi=0) .
$$

Since $\widetilde{\mathbf{W}_{N}^{1}}=0$ and $\overline{\mathbf{W}^{1}}=0$, we obtain that

$$
\mathbf{V}_{N}^{1} \equiv 0 .
$$

With (4.1.9) at order $\varepsilon^{0}$, we have

$$
-\frac{\partial U^{0}}{\partial n}+p^{0} n=-\frac{\partial \mathbf{V}^{1}}{\partial z}+q^{0} n \text { at } z=0 .
$$

Taking the normal part of this equation, since $\mathbf{V}^{1}$ and $\frac{\partial U^{0}}{\partial n}$ are tangential on $\Gamma$, we obtain the continuity of the pressure :

$$
q^{0}(\sigma, z=0)=p^{0}(\sigma),
$$

and with the tangential part we have :

$$
\frac{\partial \mathbf{V}^{1}}{\partial z}(\sigma, z=0)=\frac{\partial U^{0}}{\partial n}(\sigma) .
$$


Equation (4.1.3) at order $\varepsilon^{-1}$ gives $\frac{\partial^{2} \mathbf{V}^{1}}{\partial z^{2}}+\frac{\partial q^{0}}{\partial z} n=0$ on $\Gamma \times[0, \kappa]$. Taking the scalar product with $n$ since $\mathbf{V}^{1}$ is tangential, we obtain that $\frac{\partial q^{0}}{\partial z}=0$ and with (4.14) we have :

$$
q^{0}(\sigma, z)=p^{0}(\sigma) \text { on } \Gamma \times[0, \kappa]
$$

and then

$$
\frac{\partial^{2} \mathbf{V}^{1}}{\partial z^{2}}=0
$$

With (4.1.10) at order $\varepsilon^{0}$ we have

$$
\left(-\frac{\partial \mathbf{V}^{1}}{\partial z}+q^{0} n\right)(z=\kappa)=\left(-\frac{\partial \mathbf{W}^{1}}{\partial \xi}+r^{0} n\right)(\xi=0) .
$$

With the normal part we have :

$$
q^{0}=r^{0}=p^{0} \text { at the boundary, }
$$

and with the tangential part:

$$
-\frac{\partial \mathbf{V}^{1}}{\partial z}=-\frac{\partial \mathbf{W}^{1}}{\partial \xi} \text { at the boundary. }
$$

Thus with (4.14) and (4.16) we have:

$$
\frac{\partial \mathbf{V}^{1}}{\partial z}(\sigma, z)=\frac{\partial U^{0}}{\partial n}(\sigma)
$$

and with (4.18) $\frac{\partial \mathbf{W}^{1}}{\partial \xi}(x, \xi)=\frac{\partial U^{0}}{\partial n}(P(x))$. We know then that :

$$
\widetilde{\mathbf{W}^{1}}(t, x, \xi)=-\frac{\partial U^{0}}{\partial n}(t, P(x)) e^{-\xi}
$$

and using (4.14), (4.16) and (4.1.9) at order $\varepsilon^{1}$ (since $\overline{\mathbf{W}^{1}}=0$ ), we have :

$$
\mathbf{V}^{1}(t, \sigma, z)=(z-\kappa-1) \frac{\partial U^{0}}{\partial n}(t, \sigma) .
$$

We can then define $\mathbf{U}^{1}$ with (4.1.1), (4.1.2), (4.1.7) and (4.1.11) at order $\varepsilon^{1}$ :

$$
\left\{\begin{array}{l}
\frac{\partial \mathbf{U}^{1}}{\partial t}-\Delta \mathbf{U}^{1}+\left(U^{0} \cdot \nabla\right) \mathbf{U}^{1}+\left(\mathbf{U}^{1} \cdot \nabla\right) U^{0}+\nabla p^{1}=0 \text { on } \Omega_{e x t} \\
\operatorname{div} \mathbf{U}^{1}=0 \text { on } \Omega_{e x t}, \\
\mathbf{U}^{1}=0 \text { on } \partial \mathcal{O} \\
\mathbf{U}^{1}=(-\kappa-1)\left(\frac{\partial U^{0}}{\partial n}\right)_{N} \text { on } \Gamma \\
\mathbf{U}^{1}(t=0)=0 \text { on } \Omega_{e x t} .
\end{array}\right.
$$




\section{Determination of the order 2 terms}

Equation (4.1.6) at order $\varepsilon^{1}$ gives $n \cdot \frac{\partial \mathbf{W}^{2}}{\partial \xi}+\operatorname{div} \mathbf{W}^{1}=0$. Integrating this equality in the variable $\xi$ we obtain the expression of $\widetilde{\mathbf{W}_{N}^{2}}$ :

$$
\widetilde{\mathbf{W}_{N}^{2}}(t, x, \xi)=-\int_{-\infty}^{\xi} \operatorname{div} \widetilde{\mathbf{W}^{1}}(t, x, \tau) d \tau=-\operatorname{div}\left(\frac{\partial U^{0}}{\partial n}(t, P(x))\right) e^{-\xi} .
$$

Writting (4.1.5) at order $\varepsilon^{0}$ we have

$$
-\frac{\partial^{2} \mathbf{W}^{2}}{\partial \xi^{2}}-2(n \cdot \nabla) \frac{\partial \mathbf{W}^{1}}{\partial \xi}-\Delta \psi \frac{\partial \mathbf{W}^{1}}{\partial \xi}+\nabla r^{0}+\frac{\partial r^{1}}{\partial \xi} n+\mathbf{W}^{2}=0 .
$$

Taking the limit when $\xi$ tends to $+\infty$ we obtain that

$$
\nabla \overline{r^{0}}+\overline{\mathbf{W}^{2}}=0 \text {. }
$$

We remark that with (4.1.6) at order $\varepsilon^{2}$ we have $n \cdot \frac{\partial \mathbf{W}^{3}}{\partial \xi}+\operatorname{div} \mathbf{W}^{2}=0$ and taking the limit when $\xi \longrightarrow+\infty$ we obtain that :

$$
\operatorname{div} \overline{\mathbf{W}^{2}}=0 .
$$

Taking the divergence of (4.25) we have then $\Delta r^{0} \equiv 0$ and since $r^{0}=p^{0}$ at the boundary, $r^{0}$ and $\overline{\mathbf{W}^{2}}$ are totally determined by

$$
\left\{\begin{array}{l}
\Delta r^{0}=0 \text { in } \Omega_{\varepsilon}, \\
r^{0}=p^{0} \text { on } \Gamma_{\varepsilon}, \\
\overline{\mathbf{W}^{2}}=-\nabla r^{0} .
\end{array}\right.
$$

Taking the scalar product of (4.24) with $n$ we can express $\frac{\partial \widetilde{r_{1}}}{\partial \xi}$ and integrating between $+\infty$ and $\xi$ we obtain that :

$$
\widetilde{r_{1}}(t, x, \xi)=\left(\operatorname{div}\left(\frac{\partial U^{0}}{\partial n}\right)+2(n \cdot \nabla)\left(\frac{\partial U^{0}}{\partial n}\right) \cdot n\right)(t, P(x)) e^{-\xi} .
$$

With (4.1.4) at order $\varepsilon^{0}$, we have $\frac{\partial \mathbf{V}_{N}^{2}}{\partial z}+\operatorname{div}_{\Gamma} \mathbf{V}_{T}^{1}=0$.

Equation (4.1.8) at order $\varepsilon^{2}$ gives : $\mathbf{V}_{N}^{2}=\mathbf{W}_{N}^{2}$ on $\Gamma_{\varepsilon}$. In addition, $\mathbf{W}_{N}^{2}$ is totally defined and we have

$$
\mathbf{V}_{N}^{2}(t, \sigma, z=\kappa)=-\operatorname{div}\left(\frac{\partial U^{0}}{\partial n}\right)(t, \sigma)-\frac{\partial r^{0}}{\partial n}(t, \sigma+\kappa \varepsilon n(\sigma)),
$$


thus

$$
\begin{aligned}
\mathbf{V}_{N}^{2}(t, \sigma, z)= & \left(\frac{1}{2} z^{2}-(\kappa+1) z-1+\frac{1}{2} \kappa^{2}+\kappa\right) \operatorname{div}_{\Gamma}\left(\frac{\partial U^{0}}{\partial n}\right)(t, \sigma) \\
& -\frac{\partial r^{0}}{\partial n}(t, \sigma+\kappa \varepsilon n(\sigma)) .
\end{aligned}
$$

With (4.1.8) at order $\varepsilon^{1}$ we have :

$$
-\frac{\partial \mathbf{U}^{1}}{\partial n}+p^{1} n=-\frac{\partial \mathbf{V}^{2}}{\partial z}+q^{1} n \text { on } \Gamma .
$$

The normal part of this equation gives that

$$
q^{1}(t, \sigma, z=0)=-\left(\frac{\partial \mathbf{U}^{1}}{\partial n}\right)_{N}(t, \sigma)+p^{1}(t, \sigma)+(\kappa+1) \operatorname{div}_{\Gamma}\left(\frac{\partial U^{0}}{\partial n}\right)(t, \sigma) .
$$

Writing (4.1.3) at order $\varepsilon^{0}$ we obtain that :

$$
-\frac{\partial^{2} \mathbf{V}^{2}}{\partial z^{2}}-G_{0} \frac{\partial \mathbf{V}^{1}}{\partial z}+\nabla_{\Gamma_{0}} q^{0}+\frac{\partial q^{1}}{\partial z} n+\mathbf{V}^{1}=0 .
$$

The normal part of this equation gives : $\frac{\partial q^{1}}{\partial z}=\frac{\partial^{2} \mathbf{V}_{N}^{2}}{\partial z^{2}}$ and using (4.28) and (4.30) we obtain that :

$$
q^{1}(t, z, \sigma)=-\left(\frac{\partial \mathbf{U}^{1}}{\partial n}\right)_{N}(t, \sigma)+p^{1}(t, \sigma)+(\kappa+1-z) \operatorname{div}_{\Gamma}\left(\frac{\partial U^{0}}{\partial n}\right)_{T}(t, \sigma) .
$$

The tangential parts of (4.31) and (4.29) give :

$$
\begin{aligned}
\frac{\partial \mathbf{V}_{T}^{2}}{\partial z}(t, \sigma, z)= & \left(\frac{\partial \mathbf{U}^{1}}{\partial n}\right)_{T}(t, \sigma)+z G_{0}(\sigma)\left(\frac{\partial U^{0}}{\partial n}\right)_{T}(t, \sigma)-z \nabla_{\Gamma} q^{0}(t, \sigma) \\
& -\left(\frac{z^{2}}{2}-\kappa z-z\right)\left(\frac{\partial U^{0}}{\partial n}\right)_{T}(t, \sigma) .
\end{aligned}
$$

The tangential part of (4.1.10) at order $\varepsilon^{1}$ gives: $\frac{\partial \mathbf{W}_{T}^{2}}{\partial \xi}=\frac{\partial \mathbf{V}_{T}^{2}}{\partial z}$ on $\Gamma_{\varepsilon}$, thus

$$
\begin{aligned}
\frac{\partial \mathbf{W}_{T}^{2}}{\partial \xi}(t, x, \xi=0)= & \left(\frac{\partial \mathbf{U}^{1}}{\partial n}\right)_{T}(t, P(x))+\kappa G_{0}(P(x)) \frac{\partial U^{0}}{\partial n}(t, P(x)) \\
& -\kappa \nabla_{\Gamma} q^{0}(t, P(x))-\left(\kappa-\frac{\kappa^{2}}{2}\right) \frac{\partial U^{0}}{\partial n}(t, P(x)),
\end{aligned}
$$

and since with the tangential part of (4.24) we have :

$$
-\frac{\partial^{2} \widetilde{\mathbf{W}_{T}^{2}}}{\partial \xi^{2}}+\widetilde{\mathbf{W}_{T}^{2}}=2\left((n \cdot \nabla) \frac{\partial \mathbf{W}^{1}}{\partial \xi}\right)_{T}+\Delta \psi \frac{\partial \widetilde{\mathbf{W}_{T}^{1}}}{\partial \xi} .
$$


Therefore, $\mathbf{W}^{2}$ is totally determined. Integrating (4.31), $\mathbf{V}^{2}$ is totally determined. We consider then an extension of $\mathbf{V}_{\mid \Gamma}^{2}$ in $\Omega_{e x t}$ denoted by $\mathbf{U}^{2}$.

We write now the normal part of (4.1.10) at order $\varepsilon^{1}$ :

$$
-\frac{\partial \mathbf{V}_{N}^{2}}{\partial z}+q^{1}=r^{1}-\frac{\partial \mathbf{W}_{N}^{2}}{\partial \xi}-\left(\frac{\partial \mathbf{W}^{1}}{\partial n}\right)_{N} \text { on } \Gamma_{\varepsilon} .
$$

Now, with (4.20) $\frac{\partial \mathbf{W}^{1}}{\partial n}=0$, with $(4.23) \frac{\partial \mathbf{W}_{N}^{2}}{\partial \xi}(\xi=0)=\operatorname{div}\left(\frac{\partial U^{0}}{\partial n}(P(x))\right)$, and with (4.28) $\frac{\partial \mathbf{V}_{N}^{2}}{\partial z}(z=\kappa)=-\operatorname{div}_{\Gamma_{0}}\left(\frac{\partial U^{0}}{\partial n}\right)$. We obtain then using (4.32) that:

$$
r^{1}(t, x)=p^{1}(t, P(x))-\left(\frac{\partial \mathbf{U}^{1}}{\partial n}\right)_{N}(t, P(x))+\operatorname{div}\left(\frac{\partial U^{0}}{\partial n}(t, P(x))\right) \text { for } x \in \Gamma_{\varepsilon} .
$$

With (4.27) we introduce the extension $\overline{r^{1}}: \Omega_{\varepsilon} \longrightarrow \mathbb{R}$ such that

$$
\overline{r^{1}}(t, x)=p^{1}(t, P(x))-\left(\frac{\partial \mathbf{U}^{1}}{\partial n}\right)_{N}-2\left((n \cdot \nabla)\left(\frac{\partial U^{0}}{\partial n}\right)\right)_{N}(P(x)) \text { for } x \in \Gamma_{\varepsilon} .
$$

\subsection{Regularity of the profiles}

We recall that $\left(U^{0}, p^{0}\right)$ is our given regular solution of Navier Stokes Equation (1.3) with the regularity property (1.4). This $U^{0}$ is defined in the time interval $\left[0, T^{\star}\left[\right.\right.$. The existence of a regular profile $\mathbf{U}^{1}$ satisfying (4.22) is given by Proposition 3.1 replacing the coefficient $\kappa$ by $\kappa+1$.

For the other profiles, under the regularity asumptions on $U^{0}$ we have the following propositions:

Proposition 4.1 We define $\mathbf{V}^{1}$ and $\widetilde{\mathbf{W}^{1}}$ by (4.20) and (4.21).

Then for all $T<T^{*}$ and for $i=0 . .1$ we have:

$$
\left\{\begin{array}{l}
\frac{\partial^{i} \mathbf{V}^{1}}{\partial t^{i}} \in L^{\infty}\left(0, T ; H^{\frac{7}{2}-2 i}(\Gamma) \otimes \mathcal{C}^{\infty}([0, \kappa])\right) \cap L^{2}\left(0, T ; H^{\frac{9}{2}-2 i}(\Gamma) \otimes \mathcal{C}^{\infty}([0, \kappa])\right), \\
\frac{\partial^{i} \widetilde{\mathbf{W}^{1}}}{\partial t^{i}} \in L^{\infty}\left(0, T ; H^{\frac{7}{2}-2 i}\left(\Omega_{\varepsilon}\right) \otimes \mathcal{C}^{\infty}\left(\mathbb{R}^{+}\right)\right) \cap L^{2}\left(0, T ; H^{\frac{9}{2}-2 i}\left(\Omega_{\varepsilon}\right) \otimes \mathcal{C}^{\infty}\left(\mathbb{R}^{+}\right)\right)
\end{array}\right.
$$

Using Proposition 2.3 and the estimate on $p^{0}$ in (1.4) we obtain the following

Proposition 4.2 We define $r^{0}:\left[0, T^{*}\left[\times \Omega_{\varepsilon} \longrightarrow \mathbb{R}\right.\right.$ and $\overline{\mathbf{W}^{2}}:\left[0, T^{*}\left[\times \Omega_{\varepsilon} \longrightarrow \mathbb{R}^{3}\right.\right.$ by Equation (4.26). 
Then for all $T<T^{*}$, there exists a constant $C$ (which does not depend on $\varepsilon$ ) such that for $i=0 . .1$ :

$$
\left\{\begin{array}{l}
\left\|\frac{\partial^{i} r^{0}}{\partial t^{i}}\right\|_{L^{\infty}\left(0, T ; H^{4}\left(\Omega_{\varepsilon}\right)\right) \cap L^{2}\left(0, T ; H^{5}\left(\Omega_{\varepsilon}\right)\right)} \leq C, \\
\left\|\frac{\partial^{i} \overline{\mathbf{W}^{2}}}{\partial t^{i}}\right\|_{L^{\infty}\left(0, T ; H^{2}\left(\Omega_{\varepsilon}\right)\right) \cap L^{2}\left(0, T ; H^{3}\left(\Omega_{\varepsilon}\right)\right)} \leq C .
\end{array}\right.
$$

Proposition 4.3 We define $\mathbf{V}^{2}:\left[0, T^{*}\left[\times \Gamma \times[0, \kappa] \longrightarrow \mathbb{R}^{3}\right.\right.$ by (4.28) and (4.33), $\widetilde{\mathbf{W}^{2}}:\left[0, T^{*}\left[\times \Omega_{\varepsilon} \times \mathbb{R}^{+} \longrightarrow \mathbb{R}^{3}\right.\right.$ by (4.23), (4.34) and (4.35), $\widetilde{r^{1}}:\left[0, T^{*}\left[\times \Omega_{\varepsilon} \times\right.\right.$ $\mathbb{R}^{+} \longrightarrow \mathbb{R}^{3}$ by Equations (4.27) and $q^{1}:\left[0, T^{*}[\times \Gamma \times[0, \kappa] \longrightarrow \mathbb{R}\right.$ by (4.32).

Then for all $T<T^{*}$ there exists a constant $C$ such that for $i=0 . .1$,

$$
\left\{\begin{array}{l}
\left\|\frac{\partial^{i} \mathbf{V}^{2}}{\partial t^{i}}\right\|_{L^{\infty}\left(0, T ; H^{\frac{5}{2}-2 i}(\Gamma) \otimes \mathcal{C}^{\infty}([0, \kappa])\right) \cap L^{2}\left(0, T ; H^{\frac{7}{2}-2 i}(\Gamma) \otimes \mathcal{C}^{\infty}([0, \kappa])\right)} \leq C, \\
\left\|\frac{\partial^{i} \mathbf{W}^{2}}{\partial t^{i}}\right\|_{L^{\infty}\left(0, T ; H^{\frac{5}{2}-2 i}\left(\Omega_{\varepsilon}\right) \otimes \mathcal{C}^{\infty}\left(\mathbb{R}^{+}\right)\right) \cap L^{2}\left(0, T ; H^{\frac{7}{2}-2 i}\left(\Omega_{\varepsilon}\right) \otimes \mathcal{C}^{\infty}\left(\mathbb{R}^{+}\right)\right)} \leq C, \\
\left\|\widetilde{r^{1}}\right\|_{L^{\infty}\left(0, T ; H^{\frac{5}{2}}\left(\Omega_{\varepsilon}\right) \otimes \mathcal{C}^{\infty}\left(\mathbb{R}^{+}\right)\right) \cap L^{2}\left(0, T ; H^{\frac{7}{2}}\left(\Omega_{\varepsilon}\right) \otimes \mathcal{C}^{\infty}\left(\mathbb{R}^{+}\right)\right)} \leq C, \\
\left\|q^{1}\right\|_{L^{\infty}\left(0, T ; H^{\frac{5}{2}}(\Gamma) \otimes \mathcal{C}^{\infty}([0, \kappa])\right) \cap L^{2}\left(0, T ; H^{\frac{7}{2}}(\Gamma) \otimes \mathcal{C}^{\infty}([0, \kappa])\right)} \leq C .
\end{array}\right.
$$

The term $\mathbf{U}^{2}$ is an extension of $\mathbf{V}_{\mid \Gamma}^{2}$ in $\Omega_{e x t}$, then we have:

Proposition 4.4 There exists $\mathbf{U}^{2}$ satisfying $\mathbf{U}^{2}=\mathbf{V}^{2}$ on $\Gamma$ and $\operatorname{div} \mathbf{U}^{2}=0$ in $\Omega_{\text {ext }}$, such that

$$
\frac{\partial^{i} \mathbf{U}^{2}}{\partial t^{i}} \in L^{\infty}\left(0, T ; H^{3-2 i}\left(\Omega_{\text {ext }}\right)\right) \cap L^{2}\left(0, T ; H^{4-2 i}\left(\Omega_{\text {ext }}\right)\right) \text { for } i=0,1 .
$$

The inside term $\overline{r^{1}}$ is obtained by extension of a boundary value, so its regularity is deduced from classical trace relevements theorems and is described by the following

Proposition 4.5 There exists $\overline{r^{1}}:\left[0, T^{*}\left[\times \Omega_{\varepsilon} \longrightarrow \mathbb{R}\right.\right.$ satisfying (4.37) such that for all $T<T^{*}$ there exists a constant $C$ independant on $\varepsilon$ with

$$
\left\|\overline{r^{1}}\right\|_{L^{\infty}\left(0, T ; H^{3}\left(\Omega_{\varepsilon}\right)\right) \cap L^{2}\left(0, T ; H^{4}\left(\Omega_{\varepsilon}\right)\right)} \leq C .
$$




\subsection{Estimate for the remainder term}

We define $\mathbf{U}_{a}$ and $p_{a}$ the approximations of $u^{\varepsilon}$ and $p^{\varepsilon}$ given by

$$
\begin{aligned}
& \mathbf{U}_{a}(t, x)=\left\{\begin{array}{l}
U^{0}(t, x)+\varepsilon \mathbf{U}^{1}(t, x)+\varepsilon^{2} \mathbf{U}^{2}(t, x) \text { for } x \in \Omega_{e x t}, \\
\varepsilon \mathbf{V}^{1}\left(t, P(x), \frac{\varphi(x)}{\varepsilon}\right)+\varepsilon^{2} \mathbf{V}^{2}\left(t, P(x), \frac{\varphi(x)}{\varepsilon}\right) \text { for } x \in \omega_{e}, \\
\varepsilon \theta(x) \widetilde{\mathbf{W}^{1}}\left(t, x, \frac{\psi(x)}{\varepsilon}\right)+\varepsilon^{2} \theta(x) \widetilde{\mathbf{W}^{2}}\left(t, x, \frac{\psi(x)}{\varepsilon}\right) \\
\quad+\varepsilon^{2} \widetilde{\mathbf{W}^{2}}(t, x) \text { for } x \in \Omega_{\varepsilon},
\end{array}\right. \\
& p_{a}(t, x)=\left\{\begin{array}{l}
p^{0}(t, x)+\varepsilon p^{1}(t, x) \text { for } x \in \Omega_{e x t}, \\
q^{0}\left(t, P(x), \frac{\varphi(x)}{\varepsilon}\right)+\varepsilon q^{1}\left(t, P(x), \frac{\varphi(x)}{\varepsilon}\right) \text { for } x \in \omega_{\varepsilon}, \\
r^{0}(t, x)+\varepsilon \widetilde{r^{1}}\left(t, x, \frac{\psi(x)}{\varepsilon}\right)+\varepsilon \overline{r^{1}}(t, x) \text { for } x \in \Omega_{\varepsilon} .
\end{array}\right.
\end{aligned}
$$

We consider $\Psi^{\varepsilon} \in L^{\infty}\left(0, T ; H_{0}^{1}(\Omega)\right)$ satisfying

$$
\operatorname{div} \Psi^{\varepsilon}=-\frac{1}{\varepsilon^{\frac{3}{2}}} \operatorname{div} \mathbf{U}_{a}
$$

We remark that

$$
\operatorname{div} \mathbf{U}_{a}=\left\{\begin{array}{l}
\varepsilon\left(\operatorname{div}_{\Gamma_{\varphi(x)}} \mathbf{V}_{T}^{1}-\operatorname{div}_{\Gamma_{0}} \mathbf{V}_{T}^{1}\right)+\varepsilon^{2} \operatorname{div} \Gamma_{\varphi(x)} \mathbf{V}_{T}^{2} \text { if } x \in \omega_{\varepsilon}, \\
\nabla \theta \cdot\left(\widetilde{\mathbf{W}^{1}}+\varepsilon^{2} \widetilde{\mathbf{W}^{2}}\right)+\varepsilon^{2} \theta \operatorname{div} \widetilde{\mathbf{W}^{2}} \text { if } x \in \Omega_{\varepsilon} .
\end{array}\right.
$$

With the estimates performed on the different terms of the anzatz we remark that

$\left\|\frac{1}{\varepsilon^{\frac{3}{2}}} \operatorname{div} \mathbf{U}_{a}\right\|_{L^{2}(\Omega)} \leq C \varepsilon$ and then we can assume that

$$
\left\|\Psi^{\varepsilon}\right\|_{H^{1}(\Omega)}+\left\|\frac{\partial \Psi^{\varepsilon}}{\partial t}\right\|_{L^{2}(\Omega)} \leq C \varepsilon
$$

We define now $r^{\varepsilon}$ by :

$$
r^{\varepsilon}(t, x)=\left\{\begin{array}{l}
\frac{1}{\varepsilon^{\frac{3}{2}}}\left(u^{\varepsilon}(t, x)-\mathbf{U}_{a}(t, x)\right) \text { for } x \in \Omega_{e x t}, \\
\frac{1}{\varepsilon^{\frac{3}{2}}}\left(u^{\varepsilon}(t, x)-\mathbf{U}_{a}(t, x)\right)-\Psi^{\varepsilon}(t, x) \text { for } x \in \Omega .
\end{array}\right.
$$

We remark that $r^{\varepsilon} \in H_{0}^{1}(\mathcal{O})$ and $\operatorname{div} r^{\varepsilon}=0$ on $\mathcal{O}$. We take $r^{\varepsilon}$ as a test function in the weak formulation of (1.1). We obtain that :

$$
\frac{1}{2} \frac{d}{d t}\left\|r^{\varepsilon}\right\|_{L^{2}}^{2}+\left\|\nabla r^{\varepsilon}\right\|_{L^{2}}^{2}+\frac{1}{\varepsilon} \int_{\omega_{\varepsilon}}\left|r^{\varepsilon}\right|^{2}+\frac{1}{\varepsilon^{2}} \int_{\Omega_{\varepsilon}}\left|r^{\varepsilon}\right|^{2}=T_{1}+T_{2}+T_{3}+T_{4},
$$


with

$$
\begin{aligned}
T_{1}= & \frac{1}{\varepsilon^{\frac{3}{2}}}\left[\int_{\mathcal{O}} \frac{\partial \mathbf{U}_{a}}{\partial t} r^{\varepsilon}+\int_{\mathcal{O}} \nabla \mathbf{U}_{a} \nabla r^{\varepsilon}+\int_{\mathcal{O}}\left(\mathbf{U}_{a} \cdot \nabla\right) \mathbf{U}_{a} \cdot r^{\varepsilon}+\frac{1}{\varepsilon} \int_{\omega_{\varepsilon}} \mathbf{U}_{a} r^{\varepsilon}\right] \\
& +\frac{1}{\varepsilon^{\frac{3}{2}}}\left[\frac{1}{\varepsilon^{2}} \int_{\Omega_{\varepsilon}} \mathbf{U}_{a} r^{\varepsilon}+\int_{\Omega_{e x t}} f r^{\varepsilon}\right], \\
T_{2}= & \int_{\Omega} \frac{\partial \Psi^{\varepsilon}}{\partial t} r^{\varepsilon}+\int_{\Omega} \nabla \Psi^{\varepsilon} \nabla r^{\varepsilon}+\int_{\Omega}\left(\left(\Psi^{\varepsilon} \cdot \nabla\right) \mathbf{U}_{a}+\left(\mathbf{U}_{a} \cdot \nabla\right) \Psi^{\varepsilon}\right) \cdot r^{\varepsilon} \\
& +\varepsilon^{\frac{3}{2}} \int_{\Omega}\left(\Psi^{\varepsilon} \cdot \nabla\right) \Psi^{\varepsilon} \cdot r^{\varepsilon}+\frac{1}{\varepsilon} \int_{\omega_{\varepsilon}} \Psi^{\varepsilon} r^{\varepsilon}+\frac{1}{\varepsilon^{2}} \int_{\Omega_{\varepsilon}} \Psi^{\varepsilon} r^{\varepsilon}, \\
T_{3}= & -\int_{\mathcal{O}}\left(\left(r^{\varepsilon} \cdot \nabla\right)\left(\mathbf{U}_{a}+\varepsilon^{\frac{3}{2}} \Psi^{\varepsilon}\right)+\left(\left(\mathbf{U}_{a}+\varepsilon^{\frac{3}{2}} \Psi^{\varepsilon}\right) \cdot \nabla\right) r^{\varepsilon}\right) \cdot r^{\varepsilon}, \\
T_{4}= & -\varepsilon^{\frac{3}{2}} \int_{\mathcal{O}}\left(r^{\varepsilon} \cdot \nabla\right) r^{\varepsilon} \cdot r^{\varepsilon} .
\end{aligned}
$$

Lemma 4.1 For $\eta>0$, there exists a constant $C(\eta)$ such that

$$
\left|T_{1}\right| \leq \eta\left\|\nabla r^{\varepsilon}\right\|_{L^{2}}^{2}+\eta \frac{1}{\varepsilon^{2}}\left\|r^{\varepsilon}\right\|_{L^{2}\left(\Omega_{\varepsilon}\right)}^{2}+C(\eta)\left(1+\left\|r^{\varepsilon}\right\|_{L^{2}}^{2}\right) .
$$

Proof: Since $\operatorname{div} r^{\varepsilon}=0$ we can add to $T_{1}$ the term $-\int_{\mathcal{O}} p_{a} \operatorname{div} r^{\varepsilon}$. We split the integrals in $T_{1}$ in 3 terms writing that $\int_{\mathcal{O}}=\int_{\Omega_{e x t}}+\int_{\omega_{\varepsilon}}+\int_{\Omega_{\varepsilon}}$, and we have :

$$
T_{1}=\frac{1}{\varepsilon^{\frac{3}{2}}}\left(T_{11}+T_{12}+T_{13}\right),
$$

with

$$
\begin{aligned}
T_{11} & =\int_{\Omega_{e x t}}\left(\frac{\partial \mathbf{U}_{a}}{\partial t}+\left(\mathbf{U}_{a} \cdot \nabla\right) \mathbf{U}_{a}+f\right) \cdot r^{\varepsilon}+\int_{\Omega_{e x t}}\left(\nabla \mathbf{U}_{a} \nabla r^{\varepsilon}-p_{a} \operatorname{div} r^{\varepsilon}\right), \\
T_{12} & =\int_{\omega_{\varepsilon}}\left(\frac{\partial \mathbf{U}_{a}}{\partial t}+\left(\mathbf{U}_{a} \cdot \nabla\right) \mathbf{U}_{a}\right) \cdot r^{\varepsilon}+\frac{1}{\varepsilon} \int_{\omega_{\varepsilon}} \mathbf{U}_{a} r^{\varepsilon} \\
& +\int_{\omega_{\varepsilon}}\left(\nabla \mathbf{U}_{a} \nabla r^{\varepsilon}-p_{a} \operatorname{div} r^{\varepsilon}\right), \\
T_{13} & =\int_{\Omega_{\varepsilon}}\left(\frac{\partial \mathbf{U}_{a}}{\partial t}+\left(\mathbf{U}_{a} \cdot \nabla\right) \mathbf{U}_{a}\right) \cdot r^{\varepsilon}+\frac{1}{\varepsilon^{2}} \int_{\Omega_{\varepsilon}} \mathbf{U}_{a} r^{\varepsilon} \\
& +\int_{\Omega \varepsilon}\left(\nabla \mathbf{U}_{a} \nabla r^{\varepsilon}-p_{a} \operatorname{div} r^{\varepsilon}\right) .
\end{aligned}
$$


In each domain we integrate by part the last term.

For $T_{11}$ we obtain that

$$
\begin{aligned}
T_{11}= & \int_{\Omega_{e x t}}\left(\frac{\partial \mathbf{U}_{a}}{\partial t}-\Delta \mathbf{U}_{a}+\left(\mathbf{U}_{a} \cdot \nabla\right) \mathbf{U}_{a}+\nabla p_{a}-f\right) \cdot r^{\varepsilon} \\
& +\int_{\Gamma}\left(\frac{\partial \mathbf{U}_{a}}{\partial n}-p_{a} n\right) \cdot r^{\varepsilon} \\
= & \varepsilon^{2} \int_{\Omega_{e x t}}\left(\frac{\partial U^{2}}{\partial t}-\Delta U^{2}+\left(U^{0} \cdot \nabla\right) U^{2}+\left(\mathbf{U}^{1} \cdot \nabla\right) \mathbf{U}^{1}+\left(U^{2} \cdot \nabla\right) U^{0}\right) \cdot r^{\varepsilon} \\
& +\varepsilon^{3} \int_{\Omega_{e x t}}\left(\left(\mathbf{U}^{1} \cdot \nabla\right) U^{2}+\left(U^{2} \cdot \nabla\right) \mathbf{U}^{1}+\varepsilon\left(U^{2} \cdot \nabla\right) U^{2}\right) \cdot r^{\varepsilon} \\
& +\int_{\Gamma}\left[\left(\frac{\partial U^{0}}{\partial n}-p^{0} n\right)+\varepsilon\left(\frac{\partial \mathbf{U}^{1}}{\partial n}-p^{1} n\right)\right] \cdot r^{\varepsilon} \\
& \mathcal{O}\left(\varepsilon^{2}\right)\left\|r^{\varepsilon}\right\|_{L^{2}\left(\Omega_{e x t}\right)}+\int_{\Gamma}\left[\left(\frac{\partial U^{0}}{\partial n}-p^{0} n\right)+\varepsilon\left(\frac{\partial \mathbf{U}^{1}}{\partial n}-p^{1} n\right)\right] \cdot r^{\varepsilon}
\end{aligned}
$$

since we know from the regularity theorems proved in section 3 that for a fixed $T<T^{*}$ there exists a constant $C$ independant on $\varepsilon$ such that

$$
\begin{gathered}
\| \frac{\partial U^{2}}{\partial t}-\Delta U^{2}+\left(U^{0} \cdot \nabla\right) U^{2}+\left(\mathbf{U}^{1} \cdot \nabla\right) \mathbf{U}^{1}+\left(U^{2} \cdot \nabla\right) U^{0}+\varepsilon\left(\mathbf{U}^{1} \cdot \nabla\right) U^{2} \\
+\varepsilon\left(U^{2} \cdot \nabla\right) \mathbf{U}^{1}+\varepsilon^{2}\left(U^{2} \cdot \nabla\right) U^{2} \|_{L_{\left(0, T ; L^{2}\left(\Omega_{e x t}\right)\right)}} \leq C .
\end{gathered}
$$

For $T_{12}$ we have :

$$
\begin{aligned}
T_{12}= & \int_{\omega_{\varepsilon}}\left(\frac{\partial \mathbf{U}_{a}}{\partial t}-\Delta \mathbf{U}_{a}+\left(\mathbf{U}_{a} \cdot \nabla\right) \mathbf{U}_{a}+\nabla p_{a}+\frac{1}{\varepsilon} U_{a}\right) \cdot r^{\varepsilon} \\
& -\int_{\Gamma}\left(\frac{\partial \mathbf{U}_{a}}{\partial n}-p_{a} n\right) \cdot r^{\varepsilon}+\int_{\Gamma_{\varepsilon}}\left(\frac{\partial \mathbf{U}_{a}}{\partial n}-p_{a} n\right) \cdot r^{\varepsilon}
\end{aligned}
$$

that is, using the equations satisfied by the profiles, 


$$
\begin{aligned}
T_{12}= & \int_{\omega_{\varepsilon}}\left[\left(-G_{\varphi(x)}+G_{0}\right) \frac{\partial \mathbf{V}^{1}}{\partial z}+\left(\nabla_{\Gamma_{\varphi(x)}}-\nabla_{\Gamma_{0}}\right) q^{0}\right] \cdot r^{\varepsilon} \\
& +\varepsilon \int_{\omega_{\varepsilon}}\left(\frac{\partial \mathbf{V}^{1}}{\partial t}-\Delta_{\Gamma_{\varphi(x)}} \mathbf{V}^{1}-G_{\varphi(x)} \frac{\partial \mathbf{V}^{2}}{\partial z}+\nabla_{\Gamma_{\varphi(x)}} q^{1}+\mathbf{V}^{2}\right) \cdot r^{\varepsilon} \\
& +\varepsilon^{2} \int_{\omega_{\varepsilon}}\left(\frac{\partial \mathbf{V}^{2}}{\partial t}-\Delta_{\Gamma_{\varphi(x)}} \mathbf{V}^{2}+\left(\left(\mathbf{V}^{1}+\varepsilon \mathbf{V}^{2}\right) \cdot \nabla\right)\left(\mathbf{V}^{1}+\varepsilon \mathbf{V}^{2}\right)\right) \cdot r^{\varepsilon} \\
& -\int_{\Gamma}\left(\left(\frac{\partial \mathbf{V}^{1}}{\partial z}-q^{0} n\right)+\varepsilon\left(\frac{\partial \mathbf{V}^{2}}{\partial z}-q^{1} n\right)\right) \cdot r^{\varepsilon} \\
& +\int_{\Gamma_{\varepsilon}}\left(\left(\frac{\partial \mathbf{V}^{1}}{\partial z}-q^{0} n\right)+\varepsilon\left(\frac{\partial \mathbf{V}^{2}}{\partial z}-q^{1} n\right)\right) \cdot r^{\varepsilon} .
\end{aligned}
$$

Now we know that, since $\varphi(x) \leq \kappa \varepsilon$, there exists a constant $C$ such that for all $x \in \omega_{\varepsilon}$,

$$
\left|-G_{\varphi(x)}+G_{0}\right| \leq C \varepsilon \quad \text { and } \quad\left|\nabla_{\Gamma_{\varphi(x)}}-\nabla_{\Gamma_{0}}\right| \leq C \varepsilon
$$

Furthermore with Proposition 4.1 we know that for $T<T^{*}$ there exists $C$ such that

$$
\left\|\frac{\partial \mathbf{V}^{1}}{\partial z}\right\|_{L^{\infty}(] 0, T\left[\times \omega_{\varepsilon}\right)}+\left\|\nabla q^{0}\right\|_{L^{\infty}(] 0, T\left[\times \omega_{\varepsilon}\right)} \leq C,
$$

and since meas $\left(\omega_{\varepsilon}\right) \leq C \varepsilon$ we obtain that

$$
\left\|\left(-G_{\varphi(x)}+G_{0}\right) \frac{\partial \mathbf{V}^{1}}{\partial z}+\left(\nabla_{\Gamma_{\varphi(x)}}-\nabla_{\Gamma_{0}}\right) q^{0}\right\|_{L^{\infty}\left(0, T ; L^{2}\left(\omega_{\varepsilon}\right)\right)} \leq C \varepsilon^{\frac{3}{2}} .
$$

In the same way we know that

$$
\left\|\frac{\partial \mathbf{V}^{1}}{\partial t}-\Delta_{\Gamma_{\varphi(x)}} \mathbf{V}^{1}-G_{\varphi(x)} \frac{\partial \mathbf{V}^{2}}{\partial z}+\nabla_{\Gamma_{\varphi(x)}} q^{1}+\mathbf{V}^{2}\right\|_{L^{\infty}(] 0, T\left[\times \omega_{\varepsilon}\right)} \leq C,
$$

and thus

$$
\varepsilon\left\|\frac{\partial \mathbf{V}^{1}}{\partial t}-\Delta_{\Gamma_{\varphi(x)}} \mathbf{V}^{1}-G_{\varphi(x)} \frac{\partial \mathbf{V}^{2}}{\partial z}+\nabla_{\Gamma_{\varphi(x)}} q^{1}+\mathbf{V}^{2}\right\|_{L^{\infty}\left(0, T ; L^{2}\left(\omega_{\varepsilon}\right)\right)} \leq C \varepsilon^{\frac{3}{2}} .
$$

For the last inside term, we know that

$$
\left\|\frac{\partial \mathbf{V}^{2}}{\partial t}-\Delta_{\Gamma_{\varphi(x)}} \mathbf{V}^{2}+\left(\left(\mathbf{V}^{1}+\varepsilon \mathbf{V}^{2}\right) \cdot \nabla\right)\left(\mathbf{V}^{1}+\varepsilon \mathbf{V}^{2}\right)\right\|_{L^{\infty}\left(0, T ; L^{2}\left(\omega_{\varepsilon}\right)\right)} \leq C,
$$

so we obtain that

$$
\begin{aligned}
T_{12}= & \mathcal{O}\left(\varepsilon^{\frac{3}{2}}\right)\left\|r^{\varepsilon}\right\|_{L^{2}\left(\omega_{\varepsilon}\right)}-\int_{\Gamma}\left(\left(\frac{\partial \mathbf{V}^{1}}{\partial z}-q^{0} n\right)+\varepsilon\left(\frac{\partial \mathbf{V}^{2}}{\partial z}-q^{1} n\right)\right) \cdot r^{\varepsilon} \\
& +\int_{\Gamma_{\varepsilon}}\left(\left(\frac{\partial \mathbf{V}^{1}}{\partial z}-q^{0} n\right)+\varepsilon\left(\frac{\partial \mathbf{V}^{2}}{\partial z}-q^{1} n\right)\right) \cdot r^{\varepsilon} .
\end{aligned}
$$


For the third term we have

$$
\begin{aligned}
T_{13}= & \int_{\Omega_{\varepsilon}}\left(\frac{\partial \mathbf{U}_{a}}{\partial t}-\Delta \mathbf{U}_{a}+\left(\mathbf{U}_{a} \cdot \nabla\right) \mathbf{U}_{a}+\nabla p_{a}+\frac{1}{\varepsilon^{2}} r^{\varepsilon}\right) \cdot r^{\varepsilon} \\
& -\int_{\Gamma_{\varepsilon}}\left(\frac{\partial \mathbf{U}_{a}}{\partial n}-p_{a} n\right) \cdot r^{\varepsilon} \\
= & T_{131}+T_{132}+T_{133}+T_{134},
\end{aligned}
$$

with

$$
\begin{aligned}
T_{131}= & \varepsilon \int_{\Omega_{\varepsilon}} \theta\left(\frac{\partial \widetilde{\mathbf{W}^{1}}}{\partial t}-\Delta \widetilde{\mathbf{W}^{1}}-2(n \cdot \nabla) \frac{\partial \widetilde{\mathbf{W}^{2}}}{\partial \xi}-\Delta \psi \frac{\partial \widetilde{\mathbf{W}^{2}}}{\partial \xi}+\nabla r^{1}\right) \cdot r^{\varepsilon} \\
& +\varepsilon^{2} \int_{\Omega_{\varepsilon}} \theta\left(\frac{\partial \widetilde{\mathbf{W}^{2}}}{\partial t}-\Delta \widetilde{\mathbf{W}^{2}}\right) \cdot r^{\varepsilon} \\
& \left.+\varepsilon^{2} \int_{\Omega_{\varepsilon}}\left(\left(\theta \widetilde{\mathbf{W}^{1}}+\varepsilon \theta \widetilde{\mathbf{W}^{2}}+\widetilde{\mathbf{W}^{2}}\right) \cdot \nabla\right)\left(\theta \widetilde{\mathbf{W}^{1}}+\varepsilon \widetilde{\mathbf{W}^{2}}+\widetilde{\mathbf{W}^{2}}\right)\right) r^{\varepsilon} \\
& +\varepsilon^{2} \int_{\Omega_{\varepsilon}}\left(\frac{\partial \overline{\mathbf{W}^{2}}}{\partial t}-\Delta \widetilde{\mathbf{W}^{2}}\right) r^{\varepsilon}, \\
T_{132}= & -\int_{\Omega_{\varepsilon}}\left(\Delta \theta\left(\widetilde{\mathbf{W}^{1}}+\varepsilon^{2} \widetilde{\mathbf{W}^{2}}\right)-(\nabla \theta \cdot \nabla \varphi)\left(\widetilde{\mathbf{W}_{z}^{1}}+\varepsilon \widetilde{\mathbf{W}_{z}^{2}}\right)\right) r^{\varepsilon} \\
& -\varepsilon \int_{\Omega_{\varepsilon}}(\nabla \theta \cdot \nabla)\left(\widetilde{\mathbf{W}^{1}}+\varepsilon \widetilde{\mathbf{W}^{2}}\right) r^{\varepsilon}, \\
T_{133}= & -\int_{\Gamma_{\varepsilon}}\left(\varepsilon \frac{\partial \widetilde{\mathbf{W}^{2}}}{\partial n}+\varepsilon^{2} \frac{\partial \widetilde{\mathbf{W}^{2}}}{\partial n}\right) \cdot r^{\varepsilon}, \\
T_{134}= & -\int_{\Gamma_{\varepsilon}}\left(\left(\frac{\partial \widetilde{\mathbf{W}^{1}}}{\partial \xi}-r^{0} n\right)+\varepsilon\left(\frac{\partial \mathbf{W}^{2}}{\partial \xi}-r^{1} n\right)\right) \cdot r^{\varepsilon} .
\end{aligned}
$$

With the estimates concerning the profiles, we know that

$$
T_{131}+T_{133}=\mathcal{O}(\varepsilon)\left\|r^{\varepsilon}\right\|_{L^{2}\left(\Omega_{\varepsilon}\right)}+\mathcal{O}\left(\varepsilon^{2}\right)\left\|r^{\varepsilon}\right\|_{L^{2}\left(\Gamma_{\varepsilon}\right)} .
$$

Concerning $T_{132}$ we know that $\widetilde{\mathbf{W}^{1}}$ is exponentially decreasing in the variable $z$. Since $\nabla \theta=0$ in a neigbourhood of $\Gamma$, we obtain then that

$$
T_{132}=\mathcal{O}(\varepsilon)\left\|r^{\varepsilon}\right\|_{L^{2}\left(\Omega_{\varepsilon}\right)} .
$$


Hence we have

$$
\begin{aligned}
T_{13}= & \mathcal{O}(\varepsilon)\left\|r^{\varepsilon}\right\|_{L^{2}\left(\Omega_{\varepsilon}\right)}+\mathcal{O}\left(\varepsilon^{2}\right)\left\|r^{\varepsilon}\right\|_{L^{2}\left(\Gamma_{\varepsilon}\right)}-\int_{\Gamma_{\varepsilon}}\left(\frac{\partial \widetilde{\mathbf{W}^{1}}}{\partial \xi}-r^{0} n\right) \cdot r^{\varepsilon} \\
& -\int_{\Gamma_{\varepsilon}} \varepsilon\left(\frac{\partial \mathbf{W}^{2}}{\partial \xi}-r^{1} n\right) \cdot r^{\varepsilon} .
\end{aligned}
$$

Summing these three equalities, since the integrals on $\Gamma$ and $\Gamma_{\varepsilon}$ cancel by construction of the ansatz, we obtain that

$$
T_{1}=\mathcal{O}(1)\left\|r^{\varepsilon}\right\|_{L^{2}(\mathcal{O})}+\mathcal{O}\left(\frac{1}{\varepsilon^{\frac{1}{2}}}\right)\left\|r^{\varepsilon}\right\|_{L^{2}\left(\Omega_{\varepsilon}\right)}+\mathcal{O}\left(\varepsilon^{\frac{1}{2}}\right)\left\|\nabla r^{\varepsilon}\right\|_{L^{2}(\mathcal{O})}^{\frac{1}{2}}\left\|r^{\varepsilon}\right\|_{L^{2}(\mathcal{O})}^{\frac{1}{2}} .
$$

We fix now $\eta>0$. We have:

$$
\left|T_{1}\right| \leq C\left\|r^{\varepsilon}\right\|_{L^{2}(\mathcal{O})}+\frac{C}{\varepsilon^{\frac{1}{2}}}\left\|r^{\varepsilon}\right\|_{L^{2}\left(\Omega_{\varepsilon}\right)}+C\left\|\nabla r^{\varepsilon}\right\|_{L^{2}(\mathcal{O})}^{\frac{1}{2}}\left\|r^{\varepsilon}\right\|_{L^{2}(\mathcal{O})}^{\frac{1}{2}},
$$

and with Young Inequality we obtain that :

$$
\left|T_{1}\right| \leq \eta \frac{1}{\varepsilon^{2}}\left\|r^{\varepsilon}\right\|_{L^{2}\left(\Omega_{\varepsilon}\right)}^{2}+\eta\left\|\nabla r^{\varepsilon}\right\|_{L^{2}(\mathcal{O})}^{2}+C(\eta)\left(1+\left\|r^{\varepsilon}\right\|_{L^{2}(\mathcal{O})}^{2}\right) .
$$

Lemma 4.2 For $\eta>0$ there exists a constant $C(\eta)$ such that

$$
\left|T_{2}\right| \leq \eta \frac{1}{\varepsilon^{2}}\left\|r^{\varepsilon}\right\|_{L^{2}\left(\Omega_{\varepsilon}\right)}^{2}+\eta\left\|\nabla r^{\varepsilon}\right\|_{L^{2}}^{2}+C(\eta)\left(1+\left\|r^{\varepsilon}\right\|_{L^{2}}^{2}\right) .
$$

Proof: we estimate $T_{2}$ on the following way:

$$
\begin{aligned}
\left|T_{2}\right| \leq & \left\|\frac{\partial \Psi_{\varepsilon}}{\partial t}\right\|_{L^{2}}\left\|r^{\varepsilon}\right\|_{L^{2}(\Omega)}+\left\|\Psi^{\varepsilon}\right\|_{H^{1}(\Omega)}\left\|\nabla r^{\varepsilon}\right\|_{L^{2}(\Omega)} \\
& +\left(\left\|\psi^{\varepsilon}\right\|_{L^{3}(\Omega)}\left\|\nabla U_{a}\right\|_{L^{2}(\Omega)}+\left\|U_{a}\right\|_{L^{3}(\Omega)}\left\|\nabla \Psi^{\varepsilon}\right\|_{L^{2}(\Omega)}\right)\left\|r^{\varepsilon}\right\|_{L^{6}(\Omega)} \\
& +\varepsilon^{\frac{3}{2}}\left\|\Psi^{\varepsilon}\right\|_{L^{3}(\Omega)}\left\|\nabla \Psi^{\varepsilon}\right\|_{L^{2}(\Omega)}\left\|r^{\varepsilon}\right\|_{L^{6}(\Omega)}+\frac{1}{\varepsilon}\left\|\Psi^{\varepsilon}\right\|_{L^{2}\left(\omega_{\varepsilon}\right)}\left\|r^{\varepsilon}\right\|_{L^{2}\left(\omega_{\varepsilon}\right)} \\
& +\frac{1}{\varepsilon^{2}}\left\|\Psi^{\varepsilon}\right\|_{L^{2}\left(\Omega_{\varepsilon}\right)}\left\|r^{\varepsilon}\right\|_{L^{2}\left(\Omega_{\varepsilon}\right)} .
\end{aligned}
$$

Using that there exists $C$ such that $\left\|\Psi^{\varepsilon}\right\|_{H^{1}(\Omega)} \leq C \varepsilon$ we obtain then that:

$$
\left|T_{2}\right| \leq C \varepsilon\left\|r^{\varepsilon}\right\|_{L^{2}(\Omega)}+C \varepsilon\left\|\nabla r^{\varepsilon}\right\|_{L^{2}(\Omega)}+C\left\|r^{\varepsilon}\right\|_{L^{2}\left(\omega_{\varepsilon}\right)}+\frac{C}{\varepsilon}\left\|r^{\varepsilon}\right\|_{L^{2}\left(\Omega_{\varepsilon}\right)} .
$$

We fix then $\eta>0$ and using Young Inequality we obtain that there exists a constant $C(\eta)$ such that

$$
\left|T_{2}\right| \leq \eta\left\|\nabla r^{\varepsilon}\right\|_{L^{2}(\mathcal{O})}^{2}+\eta \frac{1}{\varepsilon^{2}}\left\|r^{\varepsilon}\right\|_{L^{2}\left(\Omega_{\varepsilon}\right)}^{2}+C(\eta)\left(1+\left\|r^{\varepsilon}\right\|_{L^{2}(\mathcal{O})}^{2}\right) .
$$


Lemma 4.3 For $\eta>0$ there exists a constant $C(\eta)$ such that

$$
\left|T_{3}\right| \leq \eta\left\|r^{\varepsilon}\right\|_{L^{2}(\mathcal{O})}^{2}+C(\eta)\left(1+\left\|r^{\varepsilon}\right\|_{L^{2}(\mathcal{O})}^{2}\right) .
$$

Proof: we have

$$
\begin{aligned}
\left|T_{3}\right| \leq & \left\|r^{\varepsilon}\right\|_{L^{6}(\mathcal{O})}\left\|\nabla U_{a}+\varepsilon^{\frac{3}{2}} \nabla \Psi^{\varepsilon}\right\|_{L^{2}(\mathcal{O})}\left\|r^{\varepsilon}\right\|_{L^{3}(\mathcal{O})} \\
& +\left\|U_{a}+\varepsilon^{\frac{3}{2}} \Psi^{\varepsilon}\right\|_{L^{6}(\mathcal{O})}\left\|\nabla r^{\varepsilon}\right\|_{L^{2}(\mathcal{O})}\left\|r^{\varepsilon}\right\|_{L^{3}(\mathcal{O})} \\
\leq & C\left\|r^{\varepsilon}\right\|_{L^{3}(\mathcal{O})}\left\|\nabla r^{\varepsilon}\right\|_{L^{2}(\mathcal{O})} \\
\leq & C\left\|r^{\varepsilon}\right\|_{L^{2}(\mathcal{O})}\left\|\nabla r^{\varepsilon}\right\|_{L^{2}(\mathcal{O})}+C\left\|r^{\varepsilon}\right\|_{L^{2}(\mathcal{O})}^{\frac{1}{2}}\left\|\nabla r^{\varepsilon}\right\|_{L^{2}(\mathcal{O})}^{\frac{3}{2}} .
\end{aligned}
$$

We fix $\eta>0$ and using Young Inequality we obtain that there exists a constant $C(\eta)$ such that

$$
\left|T_{3}\right| \leq \eta\left\|\nabla r^{\varepsilon}\right\|_{L^{2}(\mathcal{O})}^{2}+C(\eta)\left(1+\left\|r^{\varepsilon}\right\|_{L^{2}(\mathcal{O})}^{2}\right) .
$$

\section{Lemma 4.4}

$$
T_{4}=0 .
$$

Proof: we perform the classical following computation

$$
\begin{aligned}
-\frac{1}{\varepsilon^{\frac{3}{2}}} T_{4} & =\int_{\mathcal{O}} \sum_{i, j} r_{i}^{\varepsilon} \frac{\partial r_{j}^{\varepsilon}}{\partial x_{i}} r_{j}^{\varepsilon}=\frac{1}{2} \sum_{i, j} \int_{\mathcal{O}} r_{i}^{\varepsilon} \frac{\partial}{\partial x_{i}}\left(r_{j}^{\varepsilon}\right)^{2} \\
& =-\frac{1}{2} \sum_{i, j} \int_{\mathcal{O}} \frac{\partial r_{i}^{\varepsilon}}{\partial x_{i}}\left(r_{j}^{\varepsilon}\right)^{2}=-\frac{1}{2} \int_{\mathcal{O}}\left|r^{\varepsilon}\right|^{2} \operatorname{div} r^{\varepsilon} \\
& =0 \text { since } \operatorname{div} r^{\varepsilon}=0 .
\end{aligned}
$$

Remark 4.1 The corrective term $\Psi^{\varepsilon}$ is usefull to obtain that $\operatorname{div} r^{\varepsilon}=0$. So we can use $r^{\varepsilon}$ as a test function in (1.1). In addition the divergence free property ensure that $T_{4}=0$, thus the bilinear term in the Navier-Stokes equation does not provoke a blow up for the weak solutions.

End of the proof: we take $\eta=\frac{1}{6}$ in Lemmas 4.1, 4.2 and 4.3. Adding up the obtained inequalities we obtain from (4.38) that there exists a constant $C$ such that

$$
\begin{aligned}
\frac{1}{2} \frac{d}{d t}\left\|r^{\varepsilon}\right\|_{L^{2}(\mathcal{O})}^{2}+\left\|\nabla r^{\varepsilon}\right\|_{L^{2}(\mathcal{O})}^{2}+ & \frac{1}{\varepsilon} \int_{\omega_{\varepsilon}}\left|r^{\varepsilon}\right|^{2}+\frac{1}{\varepsilon^{2}} \int_{\Omega_{\varepsilon}}\left|r^{\varepsilon}\right|^{2} \leq \frac{1}{2}\left\|\nabla r^{\varepsilon}\right\|_{L^{2}(\mathcal{O})}^{2} \\
+ & \frac{1}{2} \frac{1}{\varepsilon^{2}} \int_{\Omega_{\varepsilon}}\left|r^{\varepsilon}\right|^{2}+C\left(1+\left\|r^{\varepsilon}\right\|_{L^{2}(\mathcal{O})}^{2}\right),
\end{aligned}
$$


that is

$$
\frac{d}{d t}\left\|r^{\varepsilon}\right\|_{L^{2}(\mathcal{O})}^{2}+\left\|\nabla r^{\varepsilon}\right\|_{L^{2}(\mathcal{O})}^{2}+\frac{1}{\varepsilon} \int_{\omega_{\varepsilon}}\left|r^{\varepsilon}\right|^{2}+\frac{1}{\varepsilon^{2}} \int_{\Omega_{\varepsilon}}\left|r^{\varepsilon}\right|^{2} \leq 2 C\left(1+\left\|r^{\varepsilon}\right\|_{L^{2}(\mathcal{O})}^{2}\right),
$$

and we conlude the proof with Gronwall Lemma.

\section{References}

[1] R. A. Adams, Sobolev Spaces, Pure and Applied Mathematics, Vol. 65. Academic Press, New York-London, 1975.

[2] $\mathrm{Ph}$. Angot, Analysis of singular perturbation on the Brinkman problem for fictitious domain models of viscous flow, Math. Methods Appl. Sci. 22 (1999), 1395-1412.

[3] E. Arquis, J.P. Caltagirone, Sur les conditions hydrodynamiques au voisinage d'une interface milieu fluide-milieu poreux: application à la convection naturelle, C. R. Acad. Sci Paris, 299 Série II (1984), 1-4.

[4] F. Boyer, P. Fabrie, Eléments d'analyse pour l'étude de quelques modèles d'écoulements de fluides visqueux incompressibles, cours de D.E.A. de Mathématiques appliquées et Calcul scientifique, Université Bordeaux 1.

[5] Ch.-H. Bruneau, Numerical Simulation and Analysis of the Transition to Turbulence, Lecture Notes in Physics, 490, 1997

[6] Ch.-H. Bruneau, P. Fabrie, Effective downstream boundary conditions for incompressible navier-Stokes equations, Int. J. Numer. Methods in Fluids, 19 (1994), 693-705.

[7] Ch.-H. Bruneau, P. Fabrie, New efficient boundary conditions for incompressible navier-Stokes equations: a well-posedness result, M2AN , 30 (1996), 815-840.

[8] Ch.-H. Bruneau, I. Mortazavi, Contrôle passif d'écoulements incompressibles autour d'obstacles à l'aide de milieux poreux, C. R. Acad. Sci. Paris $\mathbf{3 2 9}$ série IIb (2001).

[9] G. Carbou, Penalization method for viscous incompressible flow around a porous thin layer, Nonlinear Anal. Real World Appl. 5 (2004), no. 5, 815855

[10] G. Carbou, P. Fabrie, Boundary layer for a penalization method for viscous incompressible flow, Adv. Differential Equations 8 (2003), no. 12, 1453-1480.

[11] D. Gilbarg, N. S. Trudinger, Elliptic partial differential equations of second order. Reprint of the 1998 edition. Classics in Mathematics. Springer-Verlag, Berlin, 2001. 
[12] V. Giraut, P. A. Raviart, Finite element methods for Navier-Stokes equations. Theory and algorithms. Springer Series in Computational Mathematics, 5. Springer-Verlag, Berlin, 1986.

[13] K. Khadra, S. Parneix, Ph. Angot, J.P. Caltagirone, Fictitious domain approach for numerical modeling of Navier-Stokes equation, Int. J. Numer. Meth. in Fluids, 34 (2000), 651-684.

[14] N. Kevlahan, J. M. Ghidaglia, Computation of turbulent flow past an array of cylinders using a spectral method with Brinkman penalization, Eur. J. Mech., B, Fluids 20 (2001), No.3, 333-350.

[15] J. Leray, Essai sur les mouvements plans d'un liquide visqueux que limitent des parois, J. Math. Pures Appli. 13 (1934), 331-418.

[16] C. Peskin, Numerical analysis of blood flow in the heart, J. Comput. Phys. 25 (1977), 220-252.

[17] C. Peskin, The fluid dynamics of heart valves: Experimental, theoretical and comptutational method, Annu. Rev. Fluid Mech 14 (1982), 235-259.

[18] E. M. Saiki, S. Biringen, Numerical simulation of a cylinder in uniform flow: application of a virtual boundary method, J. Comput. Phys. 123 (1996), 450-465.

[19] D. Sanchez, Thin Layer for Landau-Lifschitz Equation, Preprint MAB U$03.04(2003)$. 